\title{
Corela
}

Cognition, représentation, langage

4-1 | 2006

Vol. $4, n^{\circ} 1$

\section{Pour une cartographie des causatifs en anglais contemporain}

Jean-Charles Khalifa

\section{(2) OpenEdition}

\section{Journals}

Édition électronique

URL : http://journals.openedition.org/corela/429

DOI : $10.4000 /$ corela.429

ISSN : $1638-573 \mathrm{X}$

\section{Éditeur}

Cercle linguistique du Centre et de I'Ouest - CerLICO

Référence électronique

Jean-Charles Khalifa, « Pour une cartographie des causatifs en anglais contemporain », Corela [En ligne], 4-1 | 2006, mis en ligne le 28 juin 2006, consulté le 19 avril 2019. URL : http:// journals.openedition.org/corela/429; DOI : 10.4000/corela.429

Ce document a été généré automatiquement le 19 avril 2019

\section{(c) (i) (2)(2)}

Corela - cognition, représentation, langage est mis à disposition selon les termes de la licence Creative Commons Attribution - Pas d'Utilisation Commerciale - Partage dans les Mêmes Conditions 4.0 International. 


\title{
Pour une cartographie des causatifs en anglais contemporain
}

\author{
Jean-Charles Khalifa
}

Who killed Davey Moore, Why an' what's the reason for?

"Not I," says the referee,

"Don't point your finger at me I could've stopped it in the eighth

An' maybe kept him from his fate, But the crowd would've booed, I'm sure,

At not gettin' their money's worth.

It's too bad he had to go,

But there was a pressure on me too, you know.

It wasn't me that made him fall.

No, you can't blame me at all."

Bob Dylan

1 On lit rarement les épigraphes, aussi rarement que les préfaces. C'est pour cela que nous souhaiterions en préambule attirer l'attention du lecteur distrait sur celle choisie pour la présente étude. Pour tous ceux qui, lecture faite, se seront peut-être interrogés sur sa raison d'être - Bob Dylan étant réputé pour un certain nombre de choses, mais à notre connaissance pas vraiment pour son apport aux linguistiques contemporaines -, disons simplement qu'il nous semblait illustrer à merveille (à sa manière, bien évidemment...) un certain nombre des interrogations millénaires des philosophes sur la notion de causalité, et celles à peine moins anciennes des grammairiens sur le codage linguistique de la causalité. Pour ceux des lecteurs qui, à ce stade, cette fois s'interrogent sur le sérieux du linguiste qui procède à de tels rapprochements, explicitons. Le fait objectif (la relation prédicative validée, si l'on préfère) dont on part est celui-ci ${ }^{1}$ :

David ('Davey') Moore, Birth: Nov. 1, 1933, Death: Mar. 23, 1963 World's featherweight boxing champion (1959-63). Cause of death: Died of head injuries suffered in championship boxing bout with Sugar Ramos on March 21, 1963 
2 Tout linguiste sait que die est un prédicat à une place. Mais toute l'interrogation va consister, d'une part à remonter les chaînes causales, et d'autre part, et corollairement, à passer de die à kill, prédicat à deux places. La remontée de l'effet à la cause, et éventuellement aux causes premières, est par excellence un problème ontologique, et la fin de Who killed Davey Moore est d'ailleurs assez révélatrice à cet égard (nous avons mis en évidence ce qui nous paraît justifier un commentaire) :

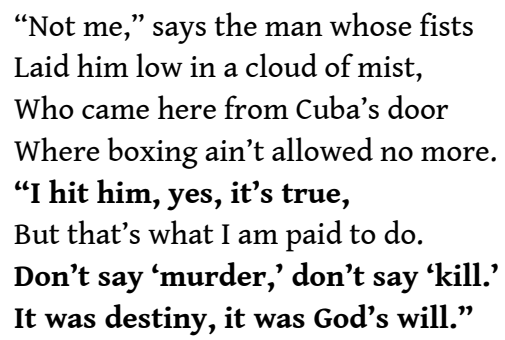

3 On observera que le recours à la cause dernière dans la dernière ligne revient, linguistiquement, à rétablir die à la place de kill; quant à l'utilisation du verbe hit, impliquant contact physique et agentivité, elle pose tout le problème de la chaîne causale et de la causation directe telle que nous aurons l'occasion de l'analyser infra. Il est tout de même assez amusant de noter que la chanson est contemporaine des premiers débats outre-Atlantique sur la décomposition lexicale, dont les exemples les plus fréquemment utilisés étaient précisément KILL > CAUSE TO DIE, et qui devaient occuper toute la décennie (voir par exemple Fodor 1970). Par ailleurs, dans le passage en épigraphe, c'est bien made him fall qui est choisi, à l'exclusion de tout autre opérateur causatif, agrammatical ou au minimum très douteux dans ce contexte (*had him fall / ??got him to fall / ?caused him to fall ). Nous tenterons dans cette étude d'apporter une contribution à l'analyse de ces questions, en posant dans un premier temps le problème de la syntaxe des constructions causatives, ce qui nous amènera directement à discuter des rôles sémantiques associés aux différentes places d'argument ; la deuxième partie décrira le système qui, selon nous, oppose les quatre opérateurs causatifs de l'anglais contemporain et les ordonne sur un schéma de quadrants définis par deux axes. Enfin, la troisième partie présente les résultats d'une étude de corpus qui nous permettra de mettre à l'épreuve des données empiriques les conclusions du modèle théorique.

\section{Syntaxe des structures causatives}

Le problème posé par ces constructions est redoutable pour le syntacticien qui voudrait à toute force faire entrer ces structures dans des catégories établies ${ }^{2}$. Comparons :

1. John wanted Mary to eat an apple

2. John forced Mary to eat an apple

3. John got Mary to eat an apple

Derrière la similitude de surface de ces énoncés, dont la structure plate serait : $\mathrm{NP}_{1} \mathrm{~V} \mathrm{NP}_{2}$ to $\mathrm{V} \mathrm{NP}_{3}$

6 on sait que l'on a affaire avec 1. à une structure dite à montée du sujet en position d'objet (subject-to-object raising) ${ }^{3}$, et avec 2., à une structure dite à contrôle par l'objet ( object control) ; examinons les analyses et tests classiques en la matière, en commençant par la première construction. 
Les verbes à montée du sujet en position d'objet

- sont de valence 2 ; parmi les tests syntaxiques qui permettent de le montrer, on retiendra les plus simples, en particulier celui de l'interrogation portant sur l'objet (What did John want?), ou celui du pseudo-clivage de ce même objet (What John wanted was...). Dans les deux cas, on constate que [Mary to eat an apple] est un constituant à part entière et se comporte comme un bloc : c'est bien le deuxième argument de WANT, verbe bivalent. On comparera force, qui ne répond pas à ces tests : *What did John force? Mary to eat an apple / What John forced was Mary to eat an apple ;

- n'assignent pas de rôle sémantique à leur objet direct apparent ; ce point se révélera crucial pour les structures causatives comme nous le verrons ultérieurement. En effet, la théorie des rôles thématiques ( $\theta$-roles) prévoit que le verbe inférieur, eat, projette deux rôles, un agent et un patient, Mary se voyant assigner le rôle d'agent, et apple celui de patient. Quant au verbe supérieur, want, il projette également deux rôles, un expérient, siège du désir, et la source de ce désir, en l'occurrence toute la proposition ${ }^{4}$ [Mary eat apple]; les quatre rôles présents dans 1 . sont bien projetés sur quatre arguments :

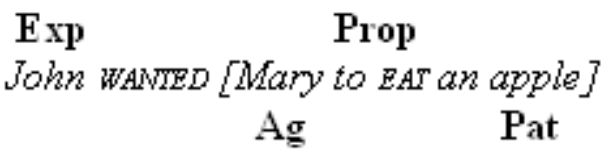

8 On comparera encore une fois utilement à la construction en force, où la situation est fort différente : ce verbe projette trois rôles, un agent, un thème (rôle proche du patient, mais sans la propriété cruciale de changement d'état ni de localisation), et une proposition. La grille- $\theta$ de eat restant bien évidemment inchangée, ce sont donc cinq ( 3 +2) rôles qui sont cette fois présents dans [2]. Or, on constate également (voir structure plate commune à [1], [2] et [3]), que nous n'avons toujours que quatre arguments. C'est une des raisons d'être, comme on le sait, de l'introduction du sujet phonologiquement nul $\mathrm{PRO}$, co-indicié à $\mathrm{NP}_{1}$ (John, le «contrôleur ", d'où le nom de ces constructions) qui va porter le rôle orphelin, en l'occurrence celui d'agent, assigné par eat. Ce qui nous donne :

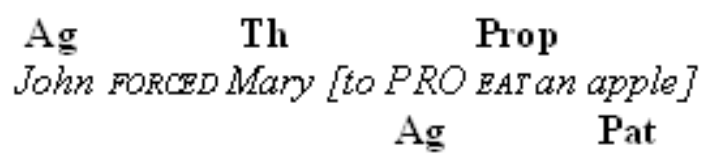

On sait par ailleurs (voir test de pronominalisation: John wanted / forced her ( ${ }^{*}$ she) to eat an apple) que, dans les deux cas, Mary se voit assigner l'accusatif par le verbe supérieur, mais avec force, cette assignation se fait classiquement dans la mesure où, pour simplifier, ce $\mathrm{NP}_{2}$ est argument de ce verbe; en revanche, avec want, nous avons affaire à un cas de marquage casuel exceptionnel (ECM: Exceptional Case Marking). Mary n'est pas un argument du verbe supérieur (dont le second argument n'est autre que la proposition entière), mais se voit tout de même marqué par ce dernier comme un objet direct ;

- n'exercent aucune contrainte de sélection sur leur objet direct apparent ; cette propriété, étroitement liée à la précédente, explique la possibilité d'avoir dans cette position des pronoms dits « explétifs », ou des NP sujets d'expressions idiomatiques :

George expected there to be some difficulty in Iraq in 2006

Arnold wanted it to rain in California very badly

George believed the shit to have hit the fan in Baghdad

Arnold found the cat to be out of the bag

${ }^{*}$ George persuaded / forced the shit to have hit the fan in Baghdad

*Arnold persuaded / forced the cat to be out of the bag 
On sait en effet que IT ou THERE ont la propriété de ne pas recevoir de rôle sémantique; c'est le cas également pour les sujets d'expressions idiomatiques (à comparer par exemple à Arnold forced the cat to get out of the bag, où l'interprétation ne peut être que référentielle : un vrai chat dans un vrai sac, cat portant dès lors tout naturellement un rôle de thème assigné par force).

Par contraste, les verbes à contrôle par l'objet (ou le sujet)

- sont de valence 3 (voir supra) ; un test utile lorsque le contexte s'y prête est la passivation de $\mathrm{NP}_{2}$ TO $\mathrm{V} \mathrm{NP}_{3}$ en $\mathrm{NP}_{3}$ TO BE $\mathrm{V}$-EN BY NP ${ }_{2}$. Les énoncés obtenus sont de valeur de vérité différente pour les constructions à contrôle, mais identique pour les constructions à montée :

John forced Mary to kiss Bill 咸 John forced Bill to be kissed by Mary

John wanted Mary to kiss Bill 㬝 John wanted Bill to be kissed by Mary

Globalement, la passivation renvoie au même événement avec les verbes de la classe want, expect, etc., mais à un événement tout autre avec ceux de la classe persuade, force..., puisque à l'actif, c'est le $\mathrm{NP}_{2}$ Mary qui subit la contrainte, mais c'est le $\mathrm{NP}_{3}$ Bill au passif ;

- assignent un rôle sémantique à leur objet direct ; d'où un autre test intéressant, la possibilité d'effacer le $3^{\mathrm{e}}$ argument (qui en l'occurrence, comme nous l'avons vu, est toute une proposition) pour obtenir un énoncé de valeur de vérité identique : John forced Mary to eat an apple $=>$ John forced Mary John wanted Mary to eat an apple $\neq>$ John wanted Mary

- exercent des contraintes de sélection sur leur objet; voir plus haut l'impossibilité d'énoncés tels *John forced it to rain / there to be a meeting, etc. ;

- forcent la co-référence entre leur objet (ou leur sujet, dans des cas très banals comme Mary tried to $P R O_{i}$ eat an apple) et le sujet (PRO) du prédicat imbriqué.

Il existe d'autres tests possibles, par exemple la possibilité de remplacer l'infinitive imbriquée soit par une complétive en THAT à temps fini, soit par un NP complexe équivalent; le test fonctionne avec les verbes à contrôle, mais est impossible avec les verbes à montée :

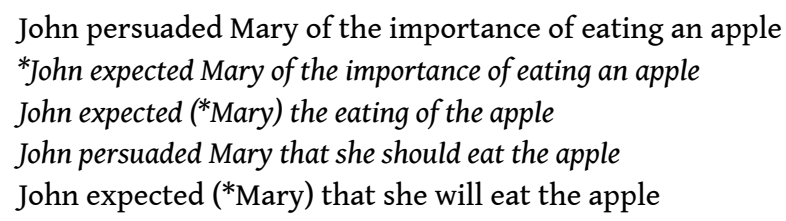

Que nous révèlent ces tests si nous les appliquons à un causatif prototypique comme [3] ? Reprenons en commençant par la fin : le NP complexe ou la complétive en THAT donnent des énoncés agrammaticaux (*John got Mary that she should eat the apple / the eating of the apple). En revanche, il est impossible d'effacer la séquence то $\mathrm{V} \mathrm{NP}_{3}\left({ }^{*} J o h n\right.$ got Mary). Les résultats sont à l'évidence contradictoires, puisque l'un est en principe révélateur d'une structure à montée, l'autre d'une structure à contrôle. Et si nous continuons à remonter la liste, nous verrons la contradiction croître et embellir, dans la mesure où 3 . réagit tantôt comme 2., tantôt comme 1. :

- Passivation : résultats mixtes, puisque l'on peut à première vue poser John got Bill to kiss Mary 國 John got Mary to be kissed by Bill ; cependant, et sous réserve d'une étude plus fine, le contraste paraît moins net qu'avec force ( $c f$. supra). D'autre part, tous les opérateurs causatifs ne présentent pas de comportement homogène vis-à-vis de ce test, car on peut tout de même envisager l'équivalence avec HAVE ou avec CAUSE, comme en témoignent ces exemples de notre corpus : 
4. The director of each society was required to submit its rules for approval to $\mathrm{Mr}$ Tidd Pratt the barrister who certified Saving Banks regulations, and afterwards to have them approved by the magistrates at quarter sessions. (B.N.C., BPH 511) [ z...and afterwards to have the magistrates at quarter sessions approve them.] 5. This fact, probably more than any other, has caused homoeopathy to be viewed with considerable scepticism by the orthodox "scientific" medical profession. (B.N.C., C9V 1135)

$[\approx$...has caused the orthodox "scientific" medical profession to view homoeopathy with considerable scepticism]

MAKE, comme on le sait, n'étant pas attestable avec une complémentation en V-en, en dehors de cas très contraints sur lesquels nous revenons infra.

- Les sujets explétifs ou les NPs sujets d'expressions idiomatiques semblent fonctionner assez bien avec les causatifs :

6. In the course of the next four years, he will continue to lead us in the right direction in hopes of establishing a "safer world and a more hopeful future." During his victory speech after being declared president for his second term, Bush said that he will attempt to earn our trust and acceptance. In an attempt to work for creating new opportunities for a higher education, Bush will make there be more chances for reading and math skills for students.

(http://www.quchronicle.com/news/2004/11/10/Opinion798696.shtml\&term)

7. I remember a doctor I worked with on sleep about a year or so ago had told me that certain medications and conditions caused there to be alpha presence. I wanted to know if anyone else has something to add to that or knows if I'm crazy or not! :-)

(http://www.sleepnet.com/tech9/messages/539.html)

8. We made the cows cannibals, an animal that normally are completely protected by it's vegetarian diet!!! Then it also show that the assumption of non transferal between species was not accurate and coupled with incubation times of around 20 years made the shit hit the fan.

(http://archive.nnytech.net/sgroup/biofuel/19738/1/)

- En revanche, interrogation comme pseudo-clivage sont rigoureusement inattestables : *What did John get / make / have / cause? Mary (to) eat an apple

*What John got / made / had / caused was Mary (to) eat an apple

14 À ce stade, on ne peut que conclure à l'ambiguïté syntaxique de la structure, qui comme on le voit possède des propriétés caractéristiques à la fois des constructions à montée et des constructions à contrôle. Autant il est tentant de gloser « $\mathrm{NP}_{1}$ est la cause de l'événement $\left[\mathrm{NP}_{2}(\mathrm{TO}) \mathrm{V} \mathrm{NP}_{3}\right]$ ", ce qui nous ferait pencher pour une structure à montée, autant il paraît difficile de soutenir que $\mathrm{NP}_{2}$ ne se voit alors pas assigner de rôle sémantique par le verbe causatif, ce qui voudrait dire en gros qu'il n'entre pas en relation avec $\mathrm{NP}_{1}$. La diachronie nous fournit en la matière un argument d'importance, mais non décisif : en vieil-anglais (Fischer 1996), les structures causatives se caractérisent par le marquage casuel par défaut du $\mathrm{NP}_{2}$, c'est-à-dire l'accusatif. En revanche, les verbes de la classe PERSUADE marquent la plupart du temps $\mathrm{NP}_{2}$ au datif.

Revenons un instant, en guise de transition entre la syntaxe et la sémantique, sur la question des rôles thématiques que nous évoquions au début de la présente étude. En matière de constructions causatives, les tentatives pour associer un rôle classique à $S_{1}$ (le sujet du verbe causatif, premier dans l'ordre linéaire) et $\mathrm{S}_{2}$ (sujet du prédicat imbriqué) ont été pour le moins problématiques. Ainsi par exemple, le point de vue ${ }^{5}$ selon lequel, dès lors que $\mathrm{S}_{1}$ est introduit dans une structure à titre d'argument supplémentaire, il devait être par défaut agent, est tout simplement contredit par les faits. On sait, même intuitivement, que s'il est confirmé par des exemples tels [9], il est infirmé par des 
exemples tout aussi nombreux tels [10], où $\mathrm{S}_{1}$, par nature inanimé, ne saurait prendre de propriétés agentives :

9. 'Maybe he wouldn't listen to the doctors. 'They'd make him listen. They'd beat him in the head, they'd lock him up in a straitjacket, they'd pump some water on him,' Santa said a little too eagerly. (John Kennedy Toole, A Confederacy of Dunces, 1980)

10. A light breeze rippled across the yard, and spikes of dusty sunshine made the trees glow, and pink and white balloons danced on their little strings. (Tim O'Brien, 'The People We Marry', in The Atlantic Monthly, January 1992)

Et que dire alors du rôle associé à $S_{2}$ ? Souvenons-nous qu'il nous est impossible de déterminer, d'un point de vue syntaxique, si ce rôle est assigné par $\mathrm{V}_{1}$ (l'opérateur causatif), ou par $V_{2}$ (le verbe de l'imbriquée), puisque nous sommes dans une construction qui possède à la fois des propriétés de la montée (assignation par $\mathrm{V}_{2}$, structure bivalente) et du contrôle (assignation par $\mathrm{V}_{1}$, structure trivalente). Doit-on conclure, en violation des principes de la théorie des rôles sémantiques (theta-criterion), soit qu'il lui est assigné par les deux, soit, ce qui ne revient pas au même mais constitue une violation tout aussi flagrante, qu'il porte deux rôles et non un seul ? Pourtant il est intuitivement tentant, dans John made Mary eat an apple ${ }^{6}$, de voir en Mary un double rôle, agent (déclencheur délibéré du procès) par rapport à eat, mais patient par rapport à make. C'est cette solution qui, dans des approches exclusivement (Jackendoff 1990) ou en partie (Alsina 1992) sémantiques, semble finalement faire consensus, à travers des appareils théoriques parfois fort élaborés. On peut toutefois se poser la question dans d'autres termes: ne serait-il pas plus économique et plus adéquat de faire des étiquettes traditionnelles de causer et causee $^{7}$, respectivement associées dans la littérature à $S_{1}$ et $S_{2}$, des rôles sémantiques à part entière ? À notre sens, ce serait là une façon de résoudre plusieurs problèmes à la fois: il suffirait de décider que seuls les quatre opérateurs causatifs assignent ces rôles, et de décrire de façon rigoureuse les primitifs sémantiques qu'ils incorporent. En particulier, on pourrait proposer, dans l'esprit des propositions de Jackendoff et d'Alsina, que le rôle assigné par le verbe inférieur est absorbé par le rôle de causativé qui prend priorité :

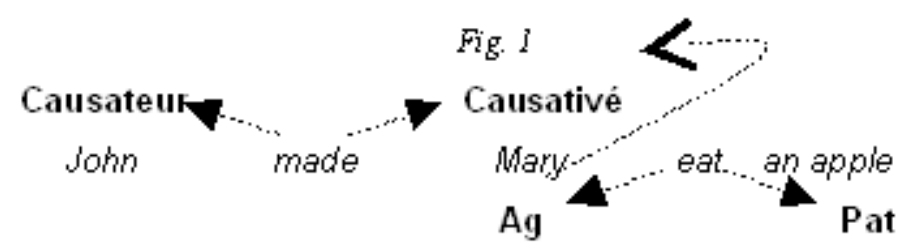

17 Nous aurons l'occasion infra de raffiner encore davantage cette présentation, et en particulier de postuler que le rôle de causateur est lui-même par nature un rôle composite ; mais passons à présent, armés de ces quelques outils, à une analyse spécifique des propriétés sémantiques des opérateurs causatifs.

\section{Les opérateurs causatifs de l'anglais : une mise en système}

18 Nous nous en tiendrons strictement au paradigme des quatre opérateurs make / have / get / cause que nous considérons comme centraux; on aura entr'aperçu au passage supra, dans la discussion de leurs propriétés syntaxiques, pourquoi nous tenons ce paradigme 
pour homogène et pourquoi nous en excluons des verbes comme force, qui par exemple permettent ( $c f$. supra) d'effacer [ rapport iconique entre la syntaxe et la sémantique, et proposons pour ce paradigme un modèle de type positionnel, une cartographie des causatifs. L'hypothèse défendue ici est que les quatre opérateurs causatifs de l'anglais forment système, un système complexe dans lequel ils s'opposent deux à deux ; bien que nous ayions été tenté dans un premier temps de mettre en avant l'analogie avec le fameux " carré des modaux ", assez séduisante il est vrai, on postulera que c'est plutôt d'un système de quadrants qu'il s'agit. Voici le schéma global dont nous partirons :

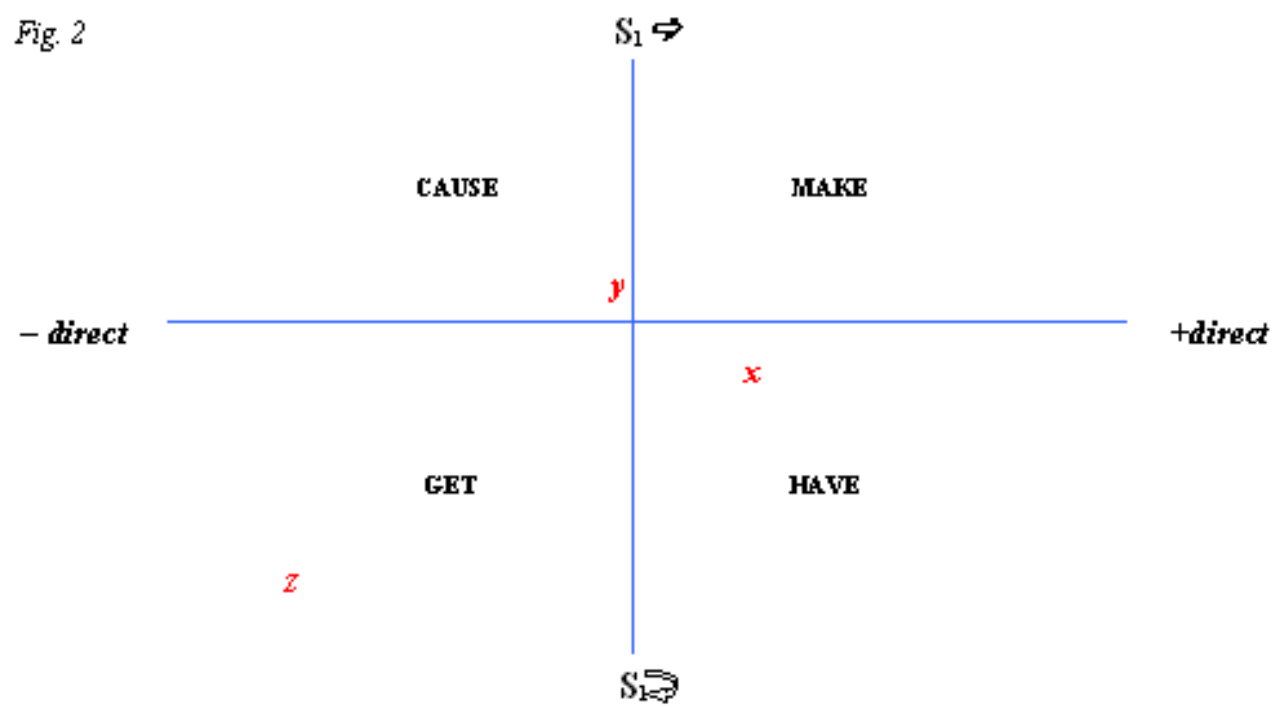

19 Dans le modèle proposé, il va de soi que, pour un énoncé donné, les valeurs obtenues en contexte peuvent se situer en n'importe quel point du quadrant occupé par l'opérateur utilisé, et que par conséquent elles peuvent se trouver très proches de la frontière d'un $(x)$ ou même plusieurs (y) quadrants, engendrant ainsi des cas d'interchangeabilité. Au contraire, elles peuvent se trouver éloignées de toute frontière $(z)$, auquel cas les substitutions deviennent impossibles, sauf à modifier radicalement tout ou partie du contexte. Il faudrait conclure, par exemple, que ce dernier cas est celui de l'énoncé cité en épigraphe, It wasn't me that made him fall / No, you can't blame me at all, où nous avons remarqué supra que les substitutions ne semblaient guère possibles. Nous allons à présent tenter de définir les deux axes qui dessinent ces quatre quadrants occupés par les quatre opérateurs causatifs de l'anglais.

\section{Axe 1 : causalité directe vs indirecte}

Ce premier axe est assez bien connu et documenté dans la littérature; il mérite cependant d'être mieux théorisé si l'on veut éviter flou et sémantique ad hoc. On peut définir ou redéfinir trois critères décisifs pour caractériser ce qui, bien entendu, n'est pas à analyser comme une opposition binaire, mais comme un gradient, bien mis en évidence par le modèle des quadrants.

- Présence d'une ou plusieurs tierces parties entre $S_{1}$ et $S_{2}$ : ce critère est le plus évident, car le plus immédiatement et intuitivement accessible. Il faut tout d'abord rappeler que les constructions causatives sont le reflet et le codage linguistique d'un schéma cognitif de base : une entité est le point de départ d'un certain flux énergétique (au sens de Talmy 2001), 
flux absorbé par une deuxième entité, et qui a pour effet de mettre cette dernière en mouvement, là où sa tendance naturelle serait le repos. On observera au passage que ce schéma est également celui de l'événement transitif prototypique : [AGENT affecte PATIENT], le premier étant vu comme point de départ, le second comme point d'arrivée. Et, de fait, nous suivrons volontiers Hopper \& Thomson 1980, Croft 1991, et bien d'autres pour voir dans la causativité l'un des paramètres centraux de la transitivité. On sait depuis l'article fondateur de Hopper \& Thompson que précisément la transitivité, en tant que faisceau de paramètres, se laisse appréhender comme un continuum sémantique au fond sans grand rapport avec la propriété purement syntaxique de transitivité du verbe, qui pour sa part est construite en tout ou rien. En l'espèce, c'est bien sur le modèle du continuum qu'il faut appréhender ce premier critère. Car dans le cas le plus simple, l'énergie circule directement de $\mathrm{S}_{1}$ jusqu'à $\mathrm{S}_{2}$, et dans les cas plus complexes, un certain nombre de relais peuvent se présenter; plus le nombre de ces relais augmente, plus la relation $\mathrm{S}_{1}-\mathrm{S}_{2}$ se verra diluée. Il nous faut, de plus, croiser ce premier critère avec les traits sémantiques associés à $S_{1}$ et $S_{2}$. Très grossièrement, dès lors que $S_{2}$ est $\{$ - animé\}, on obtiendra par défaut une interprétation de contact, de manipulation physique :

11. She made the skittle fall over

a) by pushing on it

b) by rolling the ball into it

$2111 \mathrm{~b}$ illustre le cas d'une "tierce partie » entre $S_{1}$ et $S_{2}$, empêchant donc le contact physique direct entre les deux, mais maintenant bien une relation encore directe. En revanche, si $\mathrm{S}_{1}$ et $\mathrm{S}_{2}$ sont tous deux \{+animé\}, on obtiendra des relations plus complexes ; en effet, un $\mathrm{S}_{2}$ \{+animé\} étant potentiellement agentif, il faudra également tenir compte, par exemple, de l'activation ou non-activation de cette agentivité :

12 a) She made him cough

b) She made him type the letter

On admettra sans difficulté que le sujet de $12 \mathrm{~b}$ est par nature bien plus agentif que celui de $12 \mathrm{a}$; il n'empêche que les deux énoncés sont compatibles aussi bien avec des situations où il $\mathrm{y}$ a manipulation physique (tape dans le dos pour 12a, par exemple, contrainte physique forte pour 12b) qu'avec des cas où le procès imbriqué est déclenché par des moyens beaucoup moins directs (surprise de $S_{2}$, persuasion de la part de $S_{1} \ldots$ ). En japonais, langue qui, comme on sait, a une morphologie causative productive sur le verbe, on a la possibilité d'alterner sur $\mathrm{S}_{2}$ la marque 0 de l'objet direct / patient et la marque NI de l'objet indirect ${ }^{8}$ :

(i) Mary-GA John-o ikaseta.

Mary-SUBJ John-OBJ go-CAUS-PAST

(ii) Mary-GA John-NI ikaseta.

Mary-SUBJ John-OBJ ${ }_{\text {IND }}$ go-CAUS-PAST

Il est intéressant de constater, d'ailleurs, que les linguistes japonais consultés traduisent en général (i) avec MAKE, et (ii) avec HAVE... mais cela n'infirme nullement notre raisonnement sur la causalité directe et indirecte : elle est bien directe dans les deux cas, au sens où nous la définissons ici, mais l'agentivité de $\mathrm{S}_{2}$ est supérieure d'un cran lorsque ce dernier est marqué comme objet indirect. Notre collègue T. Yamashita Smith l'exprime ainsi (c. p.) : (i) implies the intention of the causee is ignored by the causer, while in (ii) the causer typically appeals to the causee's intention to carry out the caused event.

Pour revenir aux exemples de l'anglais, dans la cartographie proposée, 11b (pour lequel il serait plus facile d'envisager une permutation avec CAUSE) serait positionné plus près de 
l'axe vertical que 11a. Quant à $12 a$ et $12 b^{9}$, ils seront, à l'évidence, plus proches de l'axe horizontal que la paire précédente; nous y reviendrons lorsque nous détaillerons cette deuxième dimension du modèle proposé, observons simplement pour le moment qu'une permutation avec HAVE est plus envisageable pour $12 \mathrm{~b}$ (She had him type the letter) que pour 12a (?She had him cough).

Fig. 3

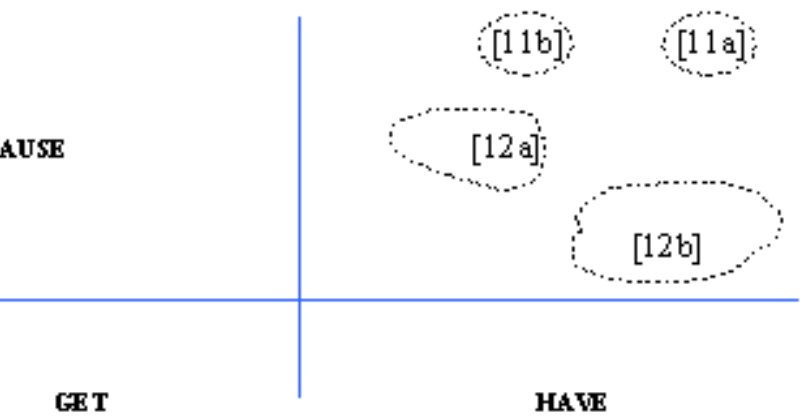

- Co-temporalité vs disjonction temporelle. Ce critère a été lui aussi assez souvent discuté dans la littérature (Givón 1993, Fischer 1990, 1995) ; il s'agit, en quelques mots, du fait que l'événement déclencheur et l'événement déclenché peuvent ou non être codés comme appartenant au même espace temporel. Même si ce concept demanderait à être affiné, même si là encore des cas-limite peuvent très certainement se présenter, on admettra sans trop de mal que plus les deux événements sont contemporains, c'est-à-dire séparés par un intervalle de temps le plus voisin possible de zéro ${ }^{10}$, plus le macro-événement est intégré et la causation interprétée par défaut comme directe. Au contraire, plus cet intervalle se distend, plus la relation de causation sera diluée, donc interprétée comme indirecte. Pour reprendre les exemples classiques, autant on peut imaginer Sugar Ramos caused Davey Moore to die on Friday by hitting him on Wednesday, autant il est strictement impossible de construire * Sugar Ramos made Davey Moore die on Friday by hitting him on Wednesday. Pour éviter des énoncés par trop artificiels, considérons :

13. In post-race interviews Dickinson emphasized the difficulties of getting a horse fit and ready for the Gold Cup, let alone winning it, and he confided that the stress and tension of training the five had caused him to lose a stone in weight since Christmas. (B.N.C., AD7 514)

14; Weekend talk of policy rifts at the highest level and of impending recession, and a perception that the Government is most reluctant to raise interest rates again, caused sterling to collapse to its lowest against the Deutschemark since the celebrated events of March last year. (B.N.C., A43 11)

15. They must've got the idea from that, called Dave Tanner and got him to set you up ...(B.N.C., H86 201)

16. Lets talk about a theme that we return to periodically, and that is propaganda and indoctrination. As a teacher, how do you get people to think for themselves? Can you in fact impart tools that will enable that? (Cobuild Corpus)

Dans ces exemples, la distance temporelle est soit explicite (since Christmas dans [13], weekend talks en [14] ${ }^{11}$ ), soit implicite (en [15], le piège, set-up, doit nécessairement être préparé et exécuté, en [16] le processus amenant à think for themselves est nécessairement long...). Les anglophones consultés pour ces quatre énoncés se déclarent unanimement très réticents à accepter une permutation en MAKE.

- Co-spatialité vs disjonction spatiale. Ce critère-là a été, à notre connaissance tout au moins, assez peu évoqué et exploré dans la littérature sur les causatifs. Il nous paraît cependant important, dans la mesure où il recoupe les propriétés sémantiques de la classe de verbes qui, diachroniquement comme synchroniquement, sont analysés comme très proches des 
causatifs, à savoir les verbes de perception ${ }^{12}$. Très schématiquement, l'événement dont $\mathrm{S}_{2}$ est le sujet est-il ou non directement observable par $\mathrm{S}_{1}$, lorsque bien entendu $\mathrm{S}_{1}$ est susceptible de pouvoir observer?

17. When John Wade was a boy of twelve, his hobby was magic. In the basement, where he practiced in front of a full-length mirror, he made his mother's silk scarves change color. He cut his father's tie with scissors and restored it whole. He placed a penny in the palm of his hand, made his hand into a fist, made the penny into a white mouse. (Tim O'Brien, 'The People we marry', in The Atlantic Monthly, Jan. 1992)

18. That was me, married to the one man who made me feel like my fiercest, most clear-hearted twelve-year-old self and not to any of the men who made me feel that other way, that euphorically grandiose, desperately insecure, wildly libidinous twenty-five-year-old way. (Lesley Dormen, 'The old economy husband', in The Atlantic Monthly, Dec. 2001)

19. He would have them sell some peculiar stock, saying: "They've given us this to get rid of, as we're the top team." (BNC, EUU 1440)

20. Shaken, Ginger bends over the tubing again. She is not changing the long snake, I discover. She is replacing only the outer section of it, the part that goes from the Clave to the junction, but I now hate having her touch me. She is contaminating me; her touch is dangerous, poison to my body. Her touch is lethal to me. I imagine ripping everything out of my arm, flinging the transparent coils away from me onto the floor. (Roxana Robinson, 'The Treatment', in The Atlantic Monthly, Sept. 2002)

Dans ces exemples, assez représentatifs dans le corpus, la condition de co-spatialité est vérifiée, soit de façon explicite (en 17, le sujet observe bien le changement de couleur, de même en 19, où manifestement would représente une modalité a posteriori), soit de façon plus subtile, comme en 18, où l'on peut dire sans trop de risque que le sujet ne ressent ce qu'elle décrit qu'en présence du mari en question. Le cas de 20 est particulier, dans la mesure (nous y reviendrons) où la dimension causative proprement dite est absente (ce n'est justement pas $S_{1}$ agissant sur $S_{2}$ ); il n'en reste pas moins que l'observation du procès imbriqué par le sujet du prédicat supérieur est obligatoire pour que l'énoncé puisse fonctionner.

On postulera que ces trois critères centraux se combinent ou s'additionnent pour définir l'axe de la causalité directe ou indirecte. Les autres paramètres qui peuvent se croiser avec ceux-là sont à notre sens davantage de l'ordre de l'inférence pragmatique ; pour

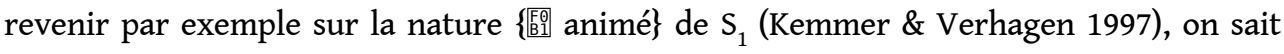
qu'il existe une corrélation assez forte entre un $\mathrm{S}_{1}$ inanimé (perçu par conséquent comme une cause et non un causateur) et la causation indirecte, et vice-versa. Cependant, comme nous l'avons vu supra et le reverrons dans bien des exemples, rien n'empêche un $\mathrm{S}_{1}$ animé de voir son agentivité neutralisée en contexte, et d'être ainsi construit comme une simple cause, dans une structure codant a priori la causation indirecte ; à l'inverse, un inanimé pourra souvent, par métaphore ou distorsion, être construit comme causateur agentif dans une structure davantage associée à la causation directe.

Notre analyse des rapports entre syntaxe des opérateurs et position sur l'axe $\mathrm{n}^{\circ} 1$ n'a rien d'original, elle demeure d'inspiration fonctionnelle-iconique, dans la lignée de Givón, Haiman, et, dans une optique diachronique, O. Fischer; nous l'avions par ailleurs développée dans Khalifa 2004. Tout se passe comme si la construction en TO $+\mathrm{V}$, caractéristique de CAUSE et GET, regroupés sur les deux quadrants à gauche du diagramme, représentait iconiquement la distance entre la cause et l'effet :

CAUSE $[\ldots . . . . . T O . . . . .$.$] EFFET$ 

bien elle qui, Samson tondu, ouvre la tente pour que ses ennemis (foomen) puissent le voir en cet état (his craft espyen). On a donc d'abord une tierce partie, donc une causation plus indirecte, codée par To $\mathrm{V}$, suivie de la construction en $\varnothing \mathrm{V}$ qui code la causation plus directe. Le premier exemple serait, sur notre système à quadrants, positionné sur la frontière, sur l'axe vertical lui-même; il est intéressant de constater, d'ailleurs, que la King James Version de la Bible utilise CAUSE: And she made him sleep upon her knees; and she called for a man, and she caused him to shave off the seven locks of his head (Judges 16:19). Et en règle générale, le modèle proposé met bien en évidence que les occurrences rencontrées peuvent se voir positionnées, au plan sémantico-pragmatique, plus ou moins près de la limite, ce qui peut entraîner des effets d'interchangeabilité. Pour illustrer ce point, reprenons l'exemple 16 supra, que nous reproduisons par commodité :

16. Lets talk about a theme that we return to periodically, and that is propaganda and indoctrination. As a teacher, how do you get people to think for themselves? Can you in fact impart tools that will enable that?

31 Nous postulerons que cet énoncé peut occuper, au plan sémantique, une zone susceptible de déborder, ne fût-ce que marginalement, sur les 3 zones voisines :

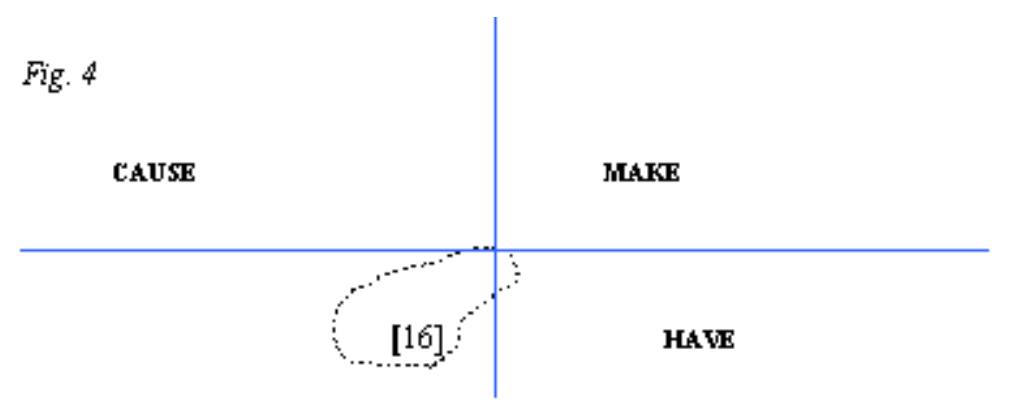

La permutation avec MAKE est en théorie possible, mais suscite beaucoup de réticences en raison des trois critères évoqués, qui en l'occurrence entrent en interaction. Si en effet on fait tendre vers zéro la distance entre la cause et l'effet, on obtient :

- un raccourcissement de la chaîne de causation: moins, voire plus du tout, de «tierces parties » entre $S_{1}$ et $S_{2}$, d'où l'effet d'une manipulation directe (critère $n^{\circ} 1$ ) ;

- une interprétation en co-temporalité (critère $n^{\circ} 2$ ), qui impliquerait que la cause (mesures prises par $\mathrm{S}_{1}$ ) et l'effet (people think for themselves) soient immédiatement consécutifs, interprétation ici absurde compte tenu des prédicats en cause, mais cependant rendue plus acceptable par la forme interrogative de l'énoncé ;

- une interprétation en co-spatialité, où $\mathrm{S}_{1}$ serait susceptible d'observer les résultats du processus de l'imbriquée (critère $\mathrm{n}^{\circ} 3$ ) ; or les résultats d'un prédicat tel think for oneself sont 
par nature difficilement observables de toutes façons, et là encore, c'est l'absence d'assertion qui rend l'énoncé moins choquant qu'à l'affirmative. amènerait un effet de sens assez curieux, celui où $\mathrm{S}_{2}$ se verrait en quelque sorte "agi sur ", contraint, là où 16 ne nous parle que de mettre en place des outils (cf. tools) pour agir non pas sur les gens eux-mêmes, mais bien plutôt sur les conditions (scolaires, etc.) dans lesquelles ils sont placés, afin d'éviter que la propagande n'ait sur eux un effet dommageable. Nous ne pouvons, à l'évidence, faute de place, détailler toutes les permutations envisageables pour chaque énoncé, mais le lecteur pourra reconstituer chaque raisonnement en reprenant les critères définis. Il est temps à présent de nous tourner vers le second axe du modèle proposé.

\section{Axe 2 : causateur affectant vs causateur affecté}

Ici, on opposera les verbes qui impliquent cognitivement une énergie unidirectionnelle partant de $S_{1}$ et allant vers $S_{2}$, à ceux qui impliquent que la même énergie revienne vers $S$ ${ }_{1}$. Plus exactement, il faudrait dire que, dans le premier cas, la focalisation est sur le trajet entre $S_{1}$ et $S_{2}$, sans que rien ne soit dit de la suite éventuelle. Dans le second cas en

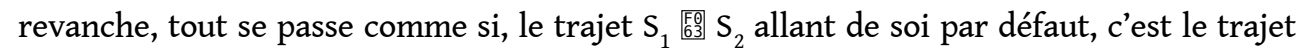
de retour vers $S_{1}$ qui devient cognitivement saillant. Le schéma abstrait d'un verbe comme HAVE ou GET suppose bel et bien que l'argument construit comme sujet syntaxique localise une entité dans sa sphère ou ramène dans sa sphère une entité initialement localisée à l'extérieur de celle-ci. Avec l'un comme l'autre, $\mathrm{S}_{1}$ est construit comme affecté (au sens de l'anglais involved) ${ }^{16}$ par la validation du procès, que cette implication donne en surface des valeurs de base telles la possession, l'acquisition, ou d'autres plus complexes et composites, comme celles que nous allons retrouver avec nos constructions causatives. Il n'empêche que, quel que soit le degré de complexité obtenu, la constante est que $\mathrm{S}_{1}$ est causateur affecté, ce qui nous amènera, au prix d'un tout relatif néologisme, de parler par opposition de causateur affectant dans l'autre cas.

Une précision s'impose à ce stade : il nous semble important de distinguer ces propriétés des rôles sémantiques classiques que sont l'agent et le bénéficiaire. Nous sommes convaincu que l'alourdissement inévitable du modèle est plus que largement compensé par un gain notable au plan théorique: en effet, un causateur affecté possède bien évidemment bon nombre des propriétés d'un bénéficiaire ${ }^{17}$, mais il est également, et dans bien des cas, sans conteste agent. On l'aura compris, HAVE et GET, dans la partie basse du diagramme, construisent par nature un causateur affecté, CAUSE et MAKE, dans la partie haute, un causateur affectant. Ces deux derniers ne disent au fond rien d'autre que le trajet de la cause à l'effet, alors que les deux premiers disent également le retour de l'effet qui devient lui-même cause pour affecter, en boucle ${ }^{18}$, le causateur-origine. On pourra très utilement à ce stade faire un détour par l'étymologie et la diachronie, qui sont à notre sens riches d'enseignements. En ce qui concerne make, on notera que, même s'il existe des désaccords entre spécialistes ${ }^{19}$, il est vraisemblablement issu de la racine indoeuropéenne *mag / mak, c'est-à-dire la même que l'on retrouve dans l'auxiliaire de modalité may. Il y a donc bien, dès l'origine, le sème de "puissance, pouvoir ", sous l'idée de "façonner ", "fabriquer ». On ne saurait mieux dire le fait que la focalisation est uniquement sur l'origine du flux énergétique, sur le mouvement de ce flux vers l'extérieur du sujet. L'étymologie de CAUSE est tout aussi intéressante, bien que 
relativement obscure ; c'est le seul opérateur des quatre qui soit d'origine latine, et il semble bien que l'origine du latin causa soit inconnue ${ }^{20}$. Cependant, le rapprochement avec « accuser » (= « mettre la cause sur »), « excuser » (= « enlever, retirer la cause »), ou encore « récuser ", montre bien ( $c f$. Robert Historique) que, là encore, c'est à une relation orientée que nous avons affaire, et ce en un double sens :

- tout d'abord, c'est bien uniquement l'origine du flux énergétique qui est focalisée, à l'exclusion du point d'arrivée, et a fortiori d'un éventuel retour vers le point-origine ;

- de plus, les premiers emplois du terme (qui sont légaux et médicaux) semblent tous converger vers une valuation négative de cette origine. De ce point de vue, il est assez spectaculaire d'interroger un corpus (le BNC, en l'espèce), et de sortir la liste des collocations cause $(\mathrm{V})+\mathrm{N}$. On obtient, dans les 30 premiers noms par ordre de fréquence : damage, problems, chaos, concern, controversy, alarm, trouble, consternation, distress, death, outrage, offence, uproar, havoc, difficulties, confusion, harassment, resentment, fire, anxiety, changes , friction, embarrassment, panic, injury, pain, loss, casualties. Doit-on commenter davantage cette liste?

Nous serons plus bref sur l'étymologie de GET et de HAVE, beaucoup plus connues, mais dont on retiendra qu'ils dérivent tous deux de racines renvoyant à la saisie d'un objet avec la main (avec des associations, entre autres, à capere > " capture » et prehendere > « appréhender ", etc.). Le mouvement d'abord en direction de la cible, puis de retour vers l'origine, est ici on ne peut plus transparent. À ce stade de l'analyse, il nous semble utile au plan théorique de faire deux rapprochements entre les schémas que nous avons dégagés et des schémas bien connus relevant $a$ priori d'autres domaines, mais qui, à notre avis, s'appliquent à plein ici.

\section{Causation, don et voix moyenne}

Si en effet on pousse un peu l'analyse, on ne peut manquer d'être frappé en premier lieu par l'étonnante ressemblance entre d'un côté les schémas cognitifs des causatifs, et de l'autre ceux associés aux constructions ditransitives, en l'espèce à GIVE et à TAKE. Sémantiquement, la constante est (voir supra) l'idée d'un flux d'énergie dont $\mathrm{S}_{1}$ est le point de départ, et dont le point d'arrivée est l'événement dont $S_{2}$ est lui-même point de départ. Dans le cas de GIVE / TAKE, la glose sera: $\mathrm{S}_{1}$ cause l'événement $\left[\mathrm{S}_{2}\right.$ entre en possession de $\mathrm{X}] /$ [ $\mathrm{S}_{1}$ entre en possession de $\mathrm{X}$ (dont $\mathrm{S}_{2}$ est dépossédé)]. On remarquera bien évidemment que la situation est sensiblement différente d'une structure transitive classique, où le deuxième participant, en tant qu'unique point d'arrivée, absorbe entièrement le flux d'énergie venant de $\mathrm{S}_{1}$, jusqu'à s'en trouver transformé (définition du patient). Dans ces conditions, un certain nombre de phénomènes se trouvent éclairés :

tout d'abord, au plan strictement syntaxique, on se souviendra que, depuis deux décennies au moins, la représentation classique des verbes à 3 arguments, dont GIVE est le prototype, passe par le double VP (VP-shell, ou « coquille de Larson »), dont nous donnons ci-dessous une représentation simplifiée ${ }^{21}$ : 


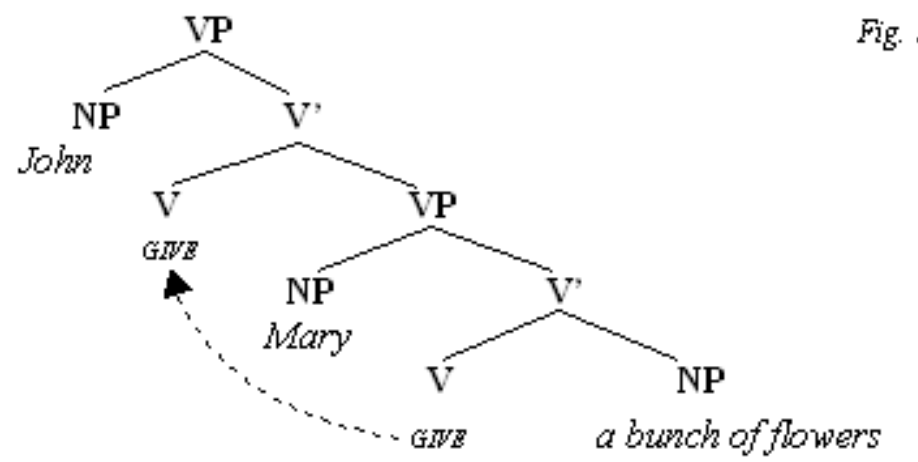

- Le diagramme est en fait un reflet assez fidèle de la décomposition sémantique donnée plus haut. Il est postulé que, sous le premier $\mathrm{V}$, se niche un opérateur causatif abstrait [CAUSE] ${ }^{22}$, qui pour être invisible n'en donne pas moins son cas au NP Mary; on lit donc : « John est la cause de l'événement (VP est complément de V) 'Mary se trouve posséder le bouquet' ». GIVE est une lexicalisation en surface de ces deux primitives CAUSE + HAVE ;

- d'autre part, on sait depuis assez longtemps (cf. entre autres Heine \& Kuteva 2001, Kemmer \& Verhagen 1997, Yamashita Smith 1998) qu'un certain nombre de langues du monde, en particulier asiatiques ${ }^{23}$ utilisent comme marqueur causatif un verbe équivalent ou dérivé de GIVE. En revanche, il est moins connu que TAKE peut également jouer ce rôle :

yígídí lá mángòrò dzú (Nupe, Niger-Congo ${ }^{24}$ )

sun took mango red

(The sun reddened the mango)

L'analogie entre l'événement causatif et l'événement ditransitif est donc patente, et on remarquera au passage que $S_{2}$ a effectivement, dans des cas comme celui-là, beaucoup plus de points communs avec un rôle sémantique but ou bénéficiaire qu'avec un rôle de patient, même si par ailleurs, il peut lui-même, selon la nature des procès en cause, se trouver affecté d'un changement d'état (exemple supra en Nupe) ; ce sera de toute façon ( cf. supra Fig.1) le rôle " causativé » qui absorbera le patient dans ce cas. Il ne nous paraît pas trop hasardeux de postuler que MAKE et CAUSE relèvent du schéma de GIVE, alors que HAVE et GET sont assimilables au schéma de TAKE. Autrement dit, dans le premier cas, il y a transfert de $\mathrm{S}_{1}$ vers $\mathrm{S}_{2}$, rien n'étant dit de l'affectation éventuelle de $\mathrm{S}_{1}$, en revanche, dans le second cas, le transfert le plus saillant cognitivement est celui qui revient vers $\mathrm{S}_{1} \cdot{ }^{25}$

Le deuxième domaine qui nous parait très clairement à rapprocher des schémas que nous avons dégagés est celui de l'opposition entre voix active et voix moyenne, très tôt décrite par les grammairiens classiques. Pour être bref et ne pas enfoncer trop de portes ouvertes, rappelons seulement que, contrairement à l'opposition actif / passif, somme toute assez minoritaire translinguistiquement, la voix moyenne n'implique pas de réorganisation en surface (remapping) des arguments, par exemple, prototypiquement :

\begin{tabular}{|c|c|}
\hline Sujet & Objet \\
\hline Agent & Patient \\
\hline \multicolumn{2}{|c|}{ Actif } \\
\hline
\end{tabular}

\begin{tabular}{|c|}
\hline Sujet \\
\hline Patient \\
\hline Passif \\
\hline
\end{tabular}

On voit que, très classiquement ici, c'est le patient qui vient occuper la position syntaxique de sujet (Bill kicked John 道 John was kicked) ; dans une voix moyenne, on aurait : 


\begin{tabular}{|c|c|}
\hline Sujet & (Objet) \\
\hline Agent / Bénéficiaire & (Patient) \\
\hline \multicolumn{2}{|c|}{ Moyen } \\
\hline
\end{tabular}

40 Nous avons mis entre parenthèses l'objet-patient pour bien montrer que la voix moyenne, qui dans les langues classiques se manifeste morphologiquement par une flexion particulière sur le verbe, peut concerner aussi bien des verbes transitifs, comme dans l'exemple ci-dessous ${ }^{26}$, en Sanskrit classique, que des verbes intransitifs ${ }^{27}$ :

devaddatạ kațam karoti

Devadatta-NOM mat-ACC makes-SG ACTIF

(Devadattah makes a mat)

devaddatạ̣ kațaṃ kurute

Devadatta-NOM mat-ACC makes-SG MOYEN

(Devadattah makes (himself) a mat)

41 Lorsque les verbes en cause ne mettent en jeu qu'un actant, on peut obtenir, très classiquement, des configurations correspondant à des réfléchis (voir le français «il se rase » ou l'anglais he's shaving Ø), ou bien des situations comme celles du grec classique :

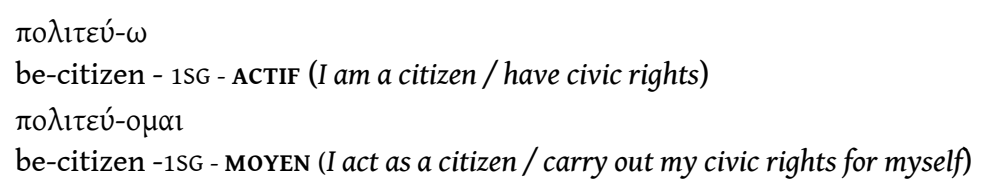

$42 \mathrm{Au}$ plan théorique, on est obligé de choisir entre deux options : soit décider (cf. notre discussion supra sur les rôles sémantiques) que le sujet syntaxique est bien porteur de deux rôles bien distincts, un rôle d'agent et un rôle de bénéficiaire ${ }^{28}$. L'autre option serait de dire que tout se passe en quelque sorte comme si (cf. diagramme supra) le second rôle était porté par un argument invisible en surface, à l'instar de PRO dans les structures à contrôle. Dans le réfléchi (Bill is washing himself vs Bill is washing his shirt), cet argument est syntaxiquement objet direct, dans les autres cas, il serait objet indirect, marqué par une préposition ( $c f$. les gloses ci-dessus). Mais dans tous les cas, ce qui est sémantiquement comme pragmatiquement marqué par la voix moyenne est ce que nous avons appelé supra l'affectation du sujet. Voici que qu'en disait J. Lyons dans un ouvrage classique :

'The implications of the middle (when it is in opposition with the active) are that the 'action' or 'state' affects the subject of the verb or his interests.' (Lyons 1968 : 373)

et, plus récemment, chez M.H. Klaiman, on trouve cette définition :

'The middle, in contrast to the active, signals the subject's coincidence with the locus of the action's principal effects.' (Klaiman 1991 : 27)

On est fort tenté de conclure, à ce stade de l'analyse, que les structures causatives en HAVE ou en GET de l'anglais contemporain fonctionnent bel et bien comme une voix moyenne, à la morphologie verbale près; ils s'opposent en cela en bloc à MAKE et CAUSE, lesquels présentent des analogies tout aussi frappantes avec la voix active. Une observation très naïve découlant de cette conclusion partielle serait la suivante : il n'est alors nullement étonnant que seuls MAKE et CAUSE puissent entrer dans des structures causatives passives. Nous avions déjà noté ce fait sans l'expliquer dans Khalifa 2004, répétons donc que, si des énoncés comme les suivants ${ }^{29}$ sont parfaitement attestés :

21. They have usually been caused to reconsider their position vis-à-vis the church through contact with a zealous Christian friend or by the church 
demonstrating a quality of life which was previously unknown to the disillusioned "back-slider". (B.N.C., CCL 1308)

22. In 1953, the year of the Coronation, we became 'New Elizabethans' (there was even a children's magazine of that name) and, sheltered from the grimmer truths of economic reality, we basked in what was made to seem like the world domination of British technology. (James Hamilton-Paterson, 'Bang', in Granta 87, 2004) des versions passives comme *Mary was had (to) eat an apple / ?*Mary was got to eat an apple. Nous éviterons d'être catégorique en ce qui concerne GET, car on arrive dans certaines circonstances à faire construire des énoncés acceptables (avec beaucoup de réticence, cependant) par certains anglophones. Il faut pour cela une modalisation de type capacité, par exemple Mary couldn't be got to eat an apple. Par ailleurs, nous n'avons trouvé dans les divers corpus fouillés que très peu (moins d'une douzaine) d'exemples authentiques de ce schéma. Certains relèvent à l'évidence d'usages figés dans des domaines techniques (fire engines / machines were got to work...); d'autres encore sont ambigus, avec une lecture possible de l'infinitive en circonstant de but, comme dans :

23. The argument is not that the check is fallible, for if it were we might still hope that enough memories could be got to prop each other up, as Ayer suggests.

(B.N.C., F9K 1333)

En tout état de cause, et sous réserve de recherches plus poussées, la passivation avec GET et HAVE va de très problématique à inattestable. On peut raisonnablement y voir un blocage dû au fait que, tout comme dans une voix moyenne, c'est $\mathrm{S}_{1}$, causateur affecté, qui est focalisé par le mouvement de retour qui le remet au premier plan. Il est tout aussi étrange de vouloir alors effacer cet argument que de vouloir passiver un énoncé à la voix moyenne.

On examinera, dans une dernière partie, comment le modèle que nous avons mis en place peut permettre de rendre compte des résultats d'une étude de corpus, et comment certains faits jusque là mal expliqués pourraient s'en trouver mieux éclairés.

\section{Apports de l'étude de corpus}

Nous avons en premier lieu travaillé sur un corpus homogène de fiction (350.000 mots), dont nous avons systématiquement extrait toutes les occurrences des schémas qui nous intéressent, en faisant tout d'abord une recherche automatisée sur concordancier ${ }^{30}$, puis en retravaillant manuellement les exemples pour écarter les $10 \%$ environ qui se sont révélés parasites. C'est ainsi que nous avons obtenu le tableau suivant :

\begin{tabular}{|c|ccc|cc|cc|}
\hline MAKE & \multicolumn{2}{|c|}{ GET } & \multicolumn{2}{c|}{ HAVE } & \multicolumn{2}{c|}{ CAUSE } \\
\hline $74,7 \%$ & $(157)$ & $14,21 \%$ & $(30)$ & $9,47 \%$ & $(20)$ & $1,89 \%$ & $(4)$ \\
\hline
\end{tabular}

Tableau $n \%$ : répartition causatifs dans un corpus homogène

On ne peut manquer d'être frappé par la dissymétrie de la répartition des opérateurs. À lui tout seul, MAKE représente les trois quarts des occurrences observées, CAUSE en revanche restant d'assez loin le plus rare. GET et HAVE quant à eux se situent, à quelques points près, au même niveau. Un premier paradoxe peut déjà se dégager, car après tout ${ }^{31}$ CAUSE est le seul de ces opérateurs qui serve exclusivement à construire des structures 
causatives, que sa complémentation soit un groupe nominal (cause chaos, death, controversy , voir supra \$2) ou une proposition. Le paradoxe se résout, toutefois, si l'on voit les choses dans l'autre sens : les structures causatives ne servent justement pas qu'à marquer que $\mathrm{A}$ est la cause de $B$, mais mettent en jeu une multitude de paramètres (affectation, volition, résistance, etc.), qui sont à divers degrés codés dans le choix des constructions et des opérateurs. Comme nous pouvons le constater sur notre modèle, CAUSE de son côté ne met en évidence que l'origine (causateur affectant), construit cette origine comme indirecte (construction en $\mathrm{TO} \mathrm{V}$ ), et accessoirement pose la conséquence comme négative pour $\mathrm{S}_{2}$. Il nous semble très significatif que l'on puisse trouver avec cet opérateur des exemples où $\mathrm{S}$ ${ }_{2}$ est directement postposé à $\mathrm{V}_{2}$ :

24. Hidden by her handmaids she is seen to blush, then turns to Actaeon and throws water in his face to blind him, to stop him seeing her naked, but that is not enough and she knows it is not enough, and soon he feels the horns growing on his forehead, dat sparso capiti vivacis cornus cervis, she caused to grow on his head the horns of the long-lived stag, as if the cost of seeing her naked had to be death, first metamorphosis then death. (B.N.C., A08 1800)

25. For Pound undoubtedly made the poem more obscure by asking for the excision of some transitional and bridging passages where the language was not at full pressure, but on the other hand he caused to be removed some extended sections which, being plainly extraneous, could only have added to readers' bafflement. (B.N.C., A1B 1722) ${ }^{32}$

Notons tout d'abord que nous déclarions cette construction strictement impossible dans Khalifa $2004^{33}$. Il est difficile de l'expliquer par des problèmes de end-weight ou de heavy NP shift, les groupes nominaux en cause étant soit relativement légers comme en [24] (the horns of the long-lived stag aurait pu sans aucun problème figurer directement à droite de CAUSE), soit effectivement lourds comme en [25] à cause de la présence de la relative, mais il était également possible d'extraposer cette dernière (he caused some extended sections to be removed which...). Il s'agit donc bien d'un choix délibéré, qui aboutit, à l'instar de $M A K E^{34}$, à mettre en contact l'opérateur causatif et $\mathrm{V}_{2}$, ce que nous analysions comme un pas vers la fusion morphologique, comme en français, où nous aurions «elle a [fait pousser] GN », «il a [fait retirer] GN ». Autrement dit, il y a bien construction d'un prédicat complexe [CAUSE-v], et on ne s'étonnera pas du fait que, dans ces conditions, seuls les opérateurs à causateur affectant puissent, même à la marge, entrer dans de tels schémas.

Nous avons tout naturellement ensuite procédé à une recherche sur chaque opérateur; comme nous avions alors besoin d'un nombre plus élevé d'occurrences, nous avons travaillé (à part pour MAKE, cf. infra et note 35) sur le British National Corpus, en rajoutant occasionnellement quelques exemples relevés manuellement au cours de nos lectures. Nous en avons tiré des tableaux que nous commenterons ci-après, en commençant par le plus fréquent des quatre : 


\begin{tabular}{|c|c|c|c|}
\hline \multicolumn{2}{|r|}{$S_{1}$} & \multicolumn{2}{|c|}{$\mathrm{S}_{2}$} \\
\hline$\{$ tanimé $\}$ & $42.7 \% \quad(67)$ & $\begin{array}{l}\{\text { tanimé }\} \\
\{\text {-animé }\}\end{array}$ & $\begin{array}{ll}67.1 \% & (45) \\
32.9 \% & (22)\end{array}$ \\
\hline f_animél & $573 \% \quad r 00$ & $\{$ tanimé\} & $73.3 \% \quad(66)$ \\
\hline & & $\{$-animé $\}$ & $26.7 \% \quad$ (24) \\
\hline
\end{tabular}

Tableau $n^{\circ} 2$ [total: 157 occurences]

51 Nous avons, dans un premier temps, étudié la répartition entre les $\mathrm{S}_{1}$ animés et inanimés ; et deux résultats importants sont aussitôt apparus, le premier et le plus évident étant que MAKE est compatible avec les deux cas de figure. On trouve aussi bien en effet des exemples du modèle de [26] que de [27] :

26. Their general morbidity made me dislike the national press screenings, so I'd often go in the evenings instead to screenings for film distributors and cinema staff, people who didn't think they'd be annihilating their critical integrity if they laughed at a gag or let out a sigh. (Andrew O'Hagan, 'Two Years in the Dark, in Granta 86, 2004)

27.I thought of the pocket money she had given me, and how my mother had taken it away because she didn't understand what good things I had done to make the house run smoothly. (Liza Ward, 'Dancing Lessons', in The Atlantic Monthly, October 2001)

Par ailleurs, il est intéressant de constater que, même si cette double compatibilité est bien connue et a été souvent relevée (Cottier 1991, Khalifa 2004), les statistiques montrent que, contrairement à une idée assez répandue, c'est bel et bien le causateur inanimé qui est majoritaire sur l'ensemble. Si nous regardons à présent du côté du causativé, cette fois le résultat s'inverse, et c'est l'animé qui est très fortement majoritaire. En croisant les deux critères, on obtient quatre cas de figure, et c'est celui où un $\mathrm{S}_{1}$ inanimé influence un $\mathrm{S}_{2}$ animé (encadré dans le tableau) qui se trouve être prototypique des emplois de MAKE causatif, donc un énoncé du type :

28. Heartland religiosity, though its fundamentalism and puritanism had often made him wince, was something Dan had been comfortable with; now it seemed barbaric. (John Updike, 'Varieties of Religious Experience', in The Atlantic Monthly, October 2002)

Nous renvoyons le lecteur à la lecture de Kemmer (op. cit. à par.) pour une étude des prédicats susceptibles d'apparaître dans l'imbriquée, dont wince est tout à fait caractéristique $^{36}$; mais notre propos n'est pas là, et nous souhaiterions revenir sur le premier point que nous avons soulevé, à savoir l'écrasante domination statistique de MAKE sur les trois autres causatifs. En anticipant quelque peu sur la fin de la présente étude, on dira que c'est en premier lieu cette propriété d'accepter tous les types de causateur qui peut l'expliquer, car nous allons rapidement voir que HAVE, GET et CAUSE sont, quant à eux, spécialisés à cet égard. Ensuite, MAKE occupe dans notre système de quadrants une position privilégiée: à la fois causation directe et causateur affectant. Or, causation directe a pour corollaire causation visible, en tous cas observable, ou en tous cas 
davantage observable que la causation indirecte, laquelle est tout naturellement associée cognitivement à l'inférence, la déduction, la supputation, etc ${ }^{37} \ldots$.. C'est au fond parce que les chaînes causales de cet ordre sont les plus simples qu'elles sont aussi les plus fréquentes. De plus, MAKE ne construit, comme nous l'avons dit à plusieurs reprises, le causateur que comme affectant, c'est-à-dire ne focalise que sur le trajet $S_{1}-S_{2}$, sans rien dire de plus sur $\mathrm{S}_{1}$. Là encore, c'est le cas le plus simple cognitivement; nous avons donc une conjonction de trois bonnes raisons qui font de make l'opérateur causatif par défaut : c'est uniquement dans les cas marqués que la relation de causation sera codée par l'un des trois autres, et ce dans des conditions que nous allons à présent détailler, en particulier en nous tournant ${ }^{38}$ vers CAUSE :

CAUSE

\begin{tabular}{|c|c|c|c|c|}
\hline \multicolumn{2}{|c|}{$S_{1}$} & TOEE V-EN & \multicolumn{2}{|c|}{$S_{2}$} \\
\hline$\{$ tanimé $\}$ & $6.6 \% \quad(31)$ & $23 \%$ (12) & $\begin{array}{l}\{\text { tanimé }\} \\
\{\text {-animé }\end{array}$ & $\begin{array}{l}74.2 \% \quad(23) \\
25.8 \% \quad(8)\end{array}$ \\
\hline \{-animé\} & $93.4 \% \quad(437)$ & $77 \% \quad(40)$ & $\begin{array}{l}\{\text { tanimé }\} \\
\{\text {-animé }\end{array}$ & \begin{tabular}{|ll}
$87.2 \%$ & $(381)$ \\
$12.8 \%$ & $(56)$
\end{tabular} \\
\hline
\end{tabular}

Tableau $n^{\circ} 3$ [total : 468 occurrences]

Ici, on remarquera d'emblée deux différences criantes avec le tableau $n^{\circ} 1:$ la répartition animé / inanimé, et la structure à imbriquée passivée. Sur le premier point, la spécialisation de CAUSE est frappante: les causateurs animés n'y représentent qu'une infime minorité, et encore, si l'on regarde de plus près certains exemples comme :

29. Then she found herself concluding that her earlier assumption had probably been correct and that there had indeed been someone in Australia, someone who had caused him to return to England in a hurry. (B.N.C., JXY 957)

30. You caused me to weep and you caused me to moan / You caused me to leave my home. (Black Girl, blues des années 20, cité dans Khalifa 2004, Ch. 5 ex. [94])

On s'aperçoit que, en dernière analyse, l'opposition animé / inanimé y est quasineutralisée, dans la mesure où on n'a pas affaire à un sujet agentif, mais à une simple cause; en reprenant nos analyses sur les rôles sémantiques (voir supra $\S 1$ ), disons que l'ingrédient presque exclusif du rôle de causateur est dans ces cas un rôle de source. En [29] comme en [30], le sujet causateur, someone / you est à la source de l'événement auquel renvoie l'imbriquée, mais uniquement par son comportement, son attitude, d'autres événements dont il aurait été l'agent, etc. On retrouve bien entendu ici l'idée de causation indirecte.

Sur le second point, la construction à imbriquée passivée est bien entendu spécifique et ne pouvait pas exister avec MAKE. Voici un exemple où l'on trouve actif et passif dans la même phrase :

31. According to Grey Walter in his book "The Living Brain" informal experiments on executed criminals showed that electric shocks caused muscles to contract and twitch, and Louis "caused an electric shock from a battery of Leyden jars to be 
administered to seven hundred Carthusian monks joined hand to hand, with prodigious effect". (B.N.C., EVA 383) beaucoup plus élevée d'animés, comme dans la $2^{\mathrm{e}}$ occurrence de l'exemple [31] ci-dessus. Nous avons dans notre corpus 52 exemples du schéma $\mathrm{S}_{1}$ CAUSE $\mathrm{S}_{2}$ TO BE V-EN, soit environ $11 \%$ du total ; mais on en compte $12 \mathrm{~S}_{1}$ animé, soit $23 \%$ de l'ensemble, à comparer aux $6,6 \%$ de la structure $S_{1}$ CAUSE $S_{2}$ V. Les animés sont donc près de 3 fois plus nombreux dans ce schéma, ce qui, même sur des chiffres absolus faibles, est suffisamment significatif (si on calcule la proportion des $\mathrm{S}_{1}$ inanimés dans ce schéma par rapport à l'ensemble des inanimés, on obtient 9,15\% ( 40 / 437), ce qui reste tout de même comparable au chiffre de 11\% mentionné supra). Observons que, dans la totalité des cas (cf. encore [31]), la raison d'être de la passivation est, très classiquement, la thématisation de l'argument patient, et la non-pertinence discursive de l'argument agent ${ }^{39}$. Il n'est sans doute pas étonnant, dans ces conditions, que la tendance soit forte de réintroduire un sujet agentif dans une configuration où aucun agent n'est présent; c'est sans doute là qu'il faut chercher l'explication de la « réintroduction de l'intentionnalité » repérée dans Cottier 19910. configuration prototypique, que nous avons encore encadrée dans le tableau, est par conséquent celle représentée par un exemple comme :

32. On the second occasion we were overcome by severe stomach pains (a touch of food poisoning, I guess), which caused us to abandon the attempt, again on Elidir Fawr - obviously our jinx mountain. (B.N.C., A15 1484)

Passons à présent de l'autre côté de l'axe horizontal, c'est-à-dire du côté des constructions à causateur affecté. Puisque nous parcourons nos quadrants dans le sens inverse des aiguilles d'une montre, nous examinerons tout d'abord GET.

GET

\begin{tabular}{|c|cc|cc|cc|c|c|}
\hline construction & \multicolumn{2}{|c|}{ To V } & \multicolumn{2}{|c|}{ V-EN } & \multicolumn{2}{c|}{ V-WG } & \multicolumn{2}{c|}{ total } \\
\hline$S_{1}\{$ tanimé $\}$ & $98.5 \%$ & $(220)$ & $86 \%$ & $(62)$ & $80 \%$ & $(24)$ & $96.4 \%$ & $(306)$ \\
\hline$S_{1}\{$-animé $\}$ & $1.5 \%$ & $(3)$ & $14 \%$ & $(10)$ & $20 \%$ & $(6)$ & $3.6 \%$ & $(19)$ \\
\hline Total & $68.8 \%$ & $(223)$ & $22 \%$ & $(72)$ & $9.2 \%$ & $(30)$ & $100 \%$ \\
\hline
\end{tabular}

Tableau $n^{\circ} 4$ [total : 325 occurrences]

On remarque ici que notre tableau se présente différemment, dans la mesure où nous trouvons trois constructions en concurrence, de distribution très inégale (dernière ligne en grisé). La construction $\mathrm{S}_{1}$ GET $\mathrm{S}_{2}$ TO $\mathrm{V}$ sera à considérer comme "non-marquée », par opposition aux constructions minoritaires et marquées $\mathrm{S}_{1}$ GET $\mathrm{S}_{2} \mathrm{~V}$-EN, et $\mathrm{S}_{1}$ GET $\mathrm{S}_{2} \mathrm{~V}$-ING, la plus rare des trois. Ce qui frappe immédiatement à la lecture du tableau est que GET semble se positionner comme le parfait complémentaire ${ }^{41}$ de CAUSE en ce qui concerne la nature du $\mathrm{S}_{1}$ : cette fois, c'est l'animé qui représente la majorité écrasante des occurrences relevées. Nous retrouverons ce phénomène avec $H A V E$, et nous pouvons anticiper quelque peu sur l'étude de cet opérateur pour rappeler qu'un causateur affecté doit tout naturellement, et par définition, être animé ; a contrario, il faut des conditions tout aussi spéciales pour que puisse apparaître un inanimé dans cette position que dans un rôle de bénéficiaire dans une construction ditransitive: \#John gave the table a book est 
sémantiquement mal formé, mais John gave the table a push est acceptable, puisque cela revient à John pushed the table, où le $2^{\mathrm{e}}$ argument est bel et bien patient et non bénéficiaire. Voici l'un des 3 exemples illustrant ce cas de figure rarissime dans notre corpus dans la construction non marquée $\mathrm{S}_{1}$ GET $\mathrm{S}_{2}$ TO V :

33. So it was the likes of that and these things that got me to know my staff and got my staff to know me. (B.N.C, K6M 647)

Le moins que l'on puisse dire, c'est que l'effet obtenu est bizarre, à tel point que certains des anglophones consultés corrigent l'énoncé pour substituer CAUSE Ou MAKE à GET... Nous avons regardé le contexte de plus près, il faut noter d'emblée que cet exemple comme les deux autres est extrait de corpus oraux; à notre sens, tout se passe comme si l'effet de sens dominant dans ces cas marginaux était celui de "surmonter un obstacle », dont on sait ${ }^{42}$ qu'il est presque toujours associé à GET. On serait assez volontiers tenté de traduire ici par « finir par », « enfin », etc. Remarquons dans notre tableau que le cas est beaucoup moins rare avec les constructions marquées, $14 \%$ pour $S_{1}$ GET $S_{2} V$-EN et jusqu'à $20 \%$ pour $S$ ${ }_{1}$ GET $\mathrm{S}_{2} \mathrm{~V}$-ING. Nous y reviendrons, mais répétons tout de même que le cas par défaut, en présence d'un causateur affecté, est celui où ce causateur est animé (humain dans la quasi-totalité des cas).

62 Les énoncés de la famille non-marquée sont tous analysables selon les deux critères définissant la position de GET dans le système. Nous comparerons simplement l'exemple [34], très représentatif de cette catégorie, à l'exemple [9] déjà cité supra, tiré du même roman, et que nous reproduisons ci-après :

34. Well, this was the last straw. Now the neighborhood was getting a bad name. She couldn't take it any more. Those people had to move. She'd get the neighbors to sign a petition. (John Kennedy Toole, A Confederacy of Dunces, 1980)

35. 'Maybe he wouldn't listen to the doctors.'

'They'd make him listen. They'd beat him in the head, they'd lock him up in a straitjacket, they'd pump some water on him,' Santa said a little too eagerly. (John Kennedy Toole, A Confederacy of Dunces, 1980)

Il est assez clair que le sujet grammatical de [34] est bien davantage affecté, au sens où, ne serait-ce que parce qu'elle l'a initié, she tire forcément un bénéfice non-nul de l'événement neighbors / sign a petition; d'autre part, la causation est à l'évidence construite comme indirecte, puisque, par exemple, la disjonction temporelle est parfaitement recevable ici (la pétition peut être signée après plusieurs heures ou plusieurs jours de réflexion). Par contraste, le sujet de [9], the doctors, est avant tout construit comme affectant, et bien entendu la causation est, en l'espèce, on ne peut plus directe (et percutante!). Même si, comme nous le posions au début de cette étude, certains exemples peuvent occuper des positions plus proches des frontières du quadrant, effaçant par là-même tel ou tel critère décisif, [34] supra est un des exemples les plus prototypiques, les plus représentatifs des relations qui se construisent avec GET.

Revenons à présent aux constructions que nous avons qualifiées de «marquées ", dont nous donnons ci-dessous deux exemples :

35. You've caught his interest and got him involved by deeds more than words.

(B.N.C., ADK 150)

36. Ashley went blue and I panicked. Jane got him breathing again. (B.N.C., K1F 2036)

La constante ici est que $\mathrm{S}_{2}$ est construit comme repéré par rapport à un état stabilisé ([35]) ou un procès dilaté ([36]) à valeur d'état temporaire ${ }^{43}$. Manipulons un peu [36], afin d'illustrer les mécanismes en jeu, pour en faire : 
36'. Ashley went blue and I panicked. Jane got him to breathe again. l'impression que $\mathrm{S}_{1}$ va quelque peu contre la volonté de $\mathrm{S}_{2}$, mais surtout, la focalisation serait sur l'entrée dans le processus breathe, et non sur la localisation de $\mathrm{S}_{2}$ dans un état, même temporaire. Il n'empêche que la constante opératoire est ailleurs : c'est bien sûr la localisation de cet état du $S_{2}$ par rapport à $S_{1}$, causateur affecté. Les primitives sémantiques constitutives de ce que nous avons nommé le rôle de causateur affecté peuvent à l'évidence se pondérer différemment en contexte, en gros, soit c'est « causateur » (agentivité, volition, etc.) qui dominera, soit ce sera " affecté » (bénéfactif, etc.) ; manifestement en [35] et [36], on est plutôt dans le premier cas, mais on trouve de nombreux exemples illustrant le deuxième, comme :

37. 'We get a lot of people knocking,' said the woman who opened the door to the house on North Bedford Drive. 'I usually don't let them in. Some of them are really crazy-people write letters to Lana Turner here saying they're in love with her, and they don't even know she's dead.' (Gaby Wood, 'In Lana Turner's Bedroom', in Granta 86, 2004)

38. I've got some notes here and I've got them covered up with something else.

(B.N.C., G3X 260)

Dans tous ces cas, le mécanisme ressemble fort à une simple thématisation de $\mathrm{S}_{1}$, dont le rôle sémantique ne peut, selon nous, qu'être celui de localisateur du bloc [S2 - VING] ; on peut bien évidemment trouver des cas intermédiaires, qui se traduiront par des positions différentes sur le quadrant, [35] et [36] par exemple se situant bien plus bas que [37] et [38]. Ceci n'a rien de très nouveau en soi et a déjà été décrit, nous n'insisterons pas davantage; redisons simplement que la pondération peut se faire soit sur les propriétés agentives, soit sur les propriétés associées à la localisation.

68 Terminons ce tour d'horizon par le dernier quadrant, celui qui définit HAVE. Regardons tout d'abord le tableau synthétique tiré de notre corpus :

HAVE

\begin{tabular}{|c|c|c|c|c|}
\hline construction & $V_{-E N}$ & V-ING & $v \varnothing$ & total \\
\hline$S_{1}\{$ tanimé $\}$ & $99.4 \% \quad(326)$ & $72.8 \% \quad(51)$ & $97.1 \% \quad(67)$ & $95 \% \quad(444)$ \\
\hline$S_{1}\{-$ animé $\}$ & $0.6 \% \quad$ (2) & $27.2 \% \quad(19)$ & $2.9 \%$ (2) & (23) \\
\hline Total & $70.23 \%$ (328) & $14.98 \%$ (70) & $14.77 \%(69)$ & $100 \%$ \\
\hline
\end{tabular}

Tableaun ${ }^{\circ}$ [total: 467 occurrences]

Ici, par position, on a donc à la fois la causation directe et un causateur affecté. Mais ce qui frappe en premier lieu à la lecture de ces données, c'est que, contrairement aux cas associés aux trois opérateurs examinés jusque là, le schéma $S_{1}$ HAVE $S_{2} V \emptyset$ non seulement n'est pas le schéma prototypique, mais est même le moins fréquent des trois attestés, à quasi-égalité avec $\mathrm{S}_{1}$ HAVE $\mathrm{S}_{2} \mathrm{~V}$-ING, et très loin (moins de $15 \%$ contre plus de $70 \%$ ) derrière $\mathrm{S}_{1}$ HAVE $\mathrm{S}_{2} \mathrm{~V}$-EN, qui dès lors devient le schéma par défaut avec HAVE. Une première explication est offerte par la diachronie : le schéma NP HAVE NP V-EN est omniprésent dès le vieil-anglais, et il est à présent généralement accepté qu'il est à l'origine du perfect de l'anglais moderne, au prix du changement dans l'ordre des mots (I have a letter written 媐 I have written a letter). En revanche, le schéma NP HAVE NP VØ est plus tardif, Visser (1963-73 : \$2069 ff) fait remonter les premiers exemples attestés à la toute fin du XIV ${ }^{\mathrm{e}}$, et 
W. Hollmann, sur la base de données encore plus étendues, note: 'I suggest that the first attested example of periphrastic causative have dates from c1440.' (2003 : 70). D'un point de vue strictement linguistique à présent, il a été très souvent souligné que HAVE n'était pas en soi un causatif, mais avait des emplois causatifs. La rareté du schéma typique des causatifs semble bien un argument supplémentaire dans ce même sens. Même si l'on regarde des exemples de ce schéma dans le corpus, on s'aperçoit qu'il est très difficile, en fait, de trouver de vraies constructions causatives au sens où nous les avons définies plus haut. Comparons :

39. Not long after Johnson saw it, a tenant farmer demolished it, wishing, perhaps, to use the stones for his own building, like the Greeks who lived near Delphi, or the old villagers of Avebury - but the landowner, presumably the same Mr Fraser, had him rebuild it. (B.N.C., G1Y 510)

40. His mother had never had him do anything like that, and while I've known women whose husbands did their own laundry and stuff like that certainly not mine. (B.N.C., EDM 1848)

41. 'When Rex lived there, he wanted me to stop talking. Now he's taking me to this party in order to have me talk. I wonder what's made him so keen on me suddenly!' (Hanif Kureishi, Gabriel's Gift, 2001)

70 Le seul véritable causatif des trois est [39], qui sur notre diagramme (Fig.1) serait positionné très près de l'axe horizontal, couvrant sans doute une zone qui déborderait sur le quadrant occupé par MAKE (la permutation HAVE / MAKE ne pose aucun problème aux anglophones consultés); on peut dire ici que les composantes agentive bénéfactive sont activées à égalité pour le causateur affecté (the landowner). En revanche, on ne peut plus tenir le même raisonnement pour [40], où la permutation avec MAKE est ressentie comme bizarre, car elle modifie le sens : le locuteur prendrait franchement parti (il faut que les mères forcent leur fils à.... $)^{44}$. Même constat en $[41]^{45}$, où il ne s'agit pas pour $\mathrm{S}_{1}($ Rex $) \mathrm{de}$ faire pression en quoi que ce soit sur $\mathrm{S}_{2}$, mais simplement de l'avoir à ses côtés pour tirer bénéfice de sa conversation brillante. La traduction de [39] ne serait pas une trahison si elle passait par "forcer à ", en revanche ce serait un faux-sens en [40] (où l'on utiliserait plutôt le neutre "faire faire »), et franchement un contresens en [41], où l'on pourrait même aller jusqu'à "pour que je fasse la conversation pour lui", en explicitant la composante bénéfactive. Et la majorité des exemples relevés est bien plutôt du type de [40] ou [41] que du type [39]. Compte tenu de la position de HAVE dans le système, la causation directe tend à peser beaucoup moins dans la balance, car comme nous l'avons $\mathrm{vu}$, ce paramètre est très largement monopolisé par MAKE, ne serait-ce que du fait de sa fréquence et de sa non-spécialisation. On peut à cet égard citer un dernier exemple tout à fait révélateur :

42. He's also the only president in 72 years to lose jobs - 1.6 million jobs lost. He's the only president to have incomes of families go down for the last three years; the only president to see exports go down; the only president to see the lowest level of business investment in our country as it is today. (John Kerry, 3rd Presidential Debate, 13/10/04, transcription intégrale Washington Post)

71 Le jeu rhétorique de John Kerry est remarquable ici pour ce qu'il révèle des mécanismes linguistiques sous-jacents. Le premier prédicat associé à son adversaire (G.W. Bush, pour ceux qui l'auraient oublié...) est lose jobs : c'est ici une sorte d'hypallage, bien évidemment Bush n'a lui-même perdu aucun emploi, en revanche il en a fait perdre aux citoyens américains. Linguistiquement, tout se passe comme si on avait affaire à un causatif lexical (le fameux KILL = CAUSE TO DIE), ce qui bien évidemment ( $c f$. Hitler killed 6 million Jews) fait porter au sujet grammatical la responsabilité aussi directe que s'il l'avait fait de ses 
propres mains ; on retrouve directement ici le problème posé par Who killed Davey Moore cité supra. Ensuite, on va jouer sur l'ambiguïté positionnelle des schémas pseudo-causatifs en HAVE: on peut comprendre aussi bien que son adversaire se voit accuser de faire délibérément baisser les revenus (agentivité), et ce en faveur (bénéfactif) de lui-même et de sa propre caste, accusation grave qu'il serait bien trop brutal de porter directement, ou bien que la baisse, tout en étant non-intentionnelle, est une conséquence mécanique de sa politique, ou bien encore qu'il est spectateur (peut-être malheureux) de cette baisse, et de ce point de vue, la juxtaposition aux deux prédicats suivants en see est tout aussi révélatrice ${ }^{46}$.

Regardons à présent du côté des deux autres schémas. On constate d'abord (cf. tableau n ${ }^{\circ}$ 5) que, avec $S_{1}$ HAVE $S_{2} V$-ING, on retrouve une proportion sensiblement plus élevée (presque un tiers) de $\mathrm{S}_{1}$ inanimés. Nous nous contenterons de ce constat pour lequel nous n'avons pas, à ce stade, d'explication convaincante. Tout se passe comme si on avait neutralisation partielle par V-ING de la composante bénéfactive sur $\mathrm{S}_{1}$. Comparons les exemples suivants :

43. She had religiously sought out the warrant officer chef every day to offer him her tail, and by the sixth day she had him purring like a kitten. (B.N.C., FPX 822) 44. He was restored: a man of twenty-six or so, wedded to a life of bargaining, whose preferred self-image had him sitting neatly and cross-legged beside some market-booth dispensing deals and judgements like a priest, implacably, too dignified to haggle with. It had him trading crackware lamps for damaskeen silver, figs for wine, wedding figurines for Roman cloth, papyrus for salt; there was no merchandise which could not be mated and transmuted in his hands. It had him envied and admired. And rich. (Jim Crace, Quarantine, 1997)

45. And how would they have known? A temptation: a breach of the rules. Never risk having your presence noted. Keep on your side of the line. (Graham Swift, The Light of Day, 2003)

46. Planning teams developing materials in a local area can have them produced on high quality equipment, and the materials can be available to others who may find them useful at minimal cost. (B.N.C., EW7 397)

73 En [43], $\mathrm{S}_{1}$ est très clairement bénéficiaire, mais la composante agentive est voisine de zéro, puisque purr like a kitten n'est pas l'effet directement recherché par le causateur. En [44], pour les deux premières occurrences, $S_{1}$, inanimé, ne peut pas être construit comme bénéficiaire, pas davantage comme agent. On est ici à nouveau dans la localisation, avec une valeur de HAVE finalement pas si éloignée de celle de possession : him sitting... / him trading...sont bel et bien des composantes, des ingrédients, donc appartiennent à cette image de soi du sujet grammatical. C'est la même valeur que l'on retrouve avec la troisième occurrence, It had him envied and admired. And rich., où les participes passés ont une valeur quasi-adjectivale (voir la coordination avec rich, mais il est impossible d'avoir *It had him rich). Ce qui nous fournit une transition idéale pour dire un mot du schéma $\mathrm{S}_{1}$ HAVE $\mathrm{S}_{2} \mathrm{~V}-E N$, que nous avons analysé comme le schéma par défaut avec HAVE. Les exemples [45] et [46] ont un point commun assez clair, c'est la composante bénéfactive dominante sur $\mathrm{S}_{1}$. Les deux causateurs sont affectés, au sens où, en [46], c'est au bénéfice des planning teams et des sociétés qui les emploient que se fera la production, et en [45], le sujet a tout intérêt à ce que presence / not be noted. Ils ne diffèrent que par le poids de la composante agentive, plus élevée dans [46], et quasi-nulle en [45]. Tous les exemples que nous avons relevés sont explicables par la pondération relative des deux composantes.

Nous voudrions terminer ce tour d'horizon par un retour sur un blocage que nous avons évoqué dans Khalifa $2004^{47}$ sans tenter de résoudre le problème qu'il soulève. Comme 
nous l'avons vu, seuls GET et HAVE peuvent être associés au schéma _- $\mathrm{S}_{2} \mathrm{~V}$-EN, ce qui ne peut relever du seul hasard: linguistiquement, comme nous le notions supra, ce qui se construit est la localisation d'un état stabilisé du $\mathrm{S}_{2}$ par rapport à $\mathrm{S}_{1}$, causateur affecté. Un causateur affectant, par définition, agit sur $\mathrm{S}_{2}$, de telle sorte que ce qui est focalisé est le déclenchement par $S_{2}$ du procès dont il est le premier argument. On ne trouvera donc, tout naturellement, ces schémas ni avec CAUSE ni avec MAKE. Pour ce qui est de CAUSE, le schéma minoritaire en $S_{1}$ CAUSE $S_{2}$ TO BE V-EN ne constitue en aucun cas un contreexemple, puisqu'il ne renvoie justement pas à un état, mais bel et bien à un processus (voir supra ex. [31]). En ce qui concerne MAKE, la manipulation conduit à des énoncés tératologiques (*He made his car washed)... sauf dans un cas, souligné par bien des auteurs :

47. That evening she went down to the restaurant off the lobby, but the room was crowded, and so noisy she couldn't make herself heard by the maitre d'. (Jim Lewis, 'Stay up Late', in Granta 86, 2004)

48. When I arrived at St Andrews everybody was standing around, and then Arnold comes out. Nobody knew him among the caddies except me. The Anderson that was waiting for Arnold was Tip Senior. He would be in his sixties by then. A real character, but getting a bit long in the tooth. I said to old Tip, "That's Arnold over there," and he made himself known to him. (B.N.C., ASA 531)

49. After 1947 Aimé Maeght in particular undertook to support Braque and make his wartime work known to the public. (B.N.C., EBU 1570)

50. Over the next few weeks Lady Maud was intensely active. [...] and she sat almost continually on committees. In particular she made her considerable presence felt on the Committee for the Preservation of the Cleene Gorge. (Tom Sharpe, Blott on the Landscape, 1975)

Ces énoncés-là sont en revanche parfaitement naturels et même relativement fréquents ; la condition de bonne formation est la co-référence entre $S_{1}$ et le sujet de surface du prédicat passif, objet profond dans la relation imbriquée, co-référence qui peut soit passer par le réfléchi comme en [47] et [48], soit par une relation de l'ordre de la métonymie ou de la synecdoque comme en [49] et [50] ${ }^{48}$. C'est cette co-référence qui, en réintroduisant l'implication du causateur, rend possible la construction : $\mathrm{S}_{1}$ à l'évidence agit pour son propre intérêt, et le réfléchi est ici une trace quasiment iconique de ce retour vers soi-même. Cette condition de co-référence est nécessaire, mais cependant non suffisante, car comme nous le soulignions (op. cit.), des énoncés comme *he made himself washed sont tout aussi monstrueux que *he made his car washed. Les verbes associés à la structure, dans tous les exemples que nous avons pu relever, sont des verbes de cognition (know est le plus fréquent), de perception (hear, see et feel), ou autres verbes psychologiques (quelques cas avec respect). Tous ceux-là ont en commun que leur argument sujet est expérient et non agent, et d'autre part que leur argument objet est source et non patient. Autrement dit, dans les exemples du type [47] à [50], contrairement à ce que peut suggérer l'ordre linéaire, $\mathrm{S}_{1}$ n'agit pas sur $\mathrm{S}_{2}$, lequel dans ces conditions (cf. supra fig. 1) ne peut pas légitimement être analysé comme causativé. Le rôle sémantique associé à ce $\mathrm{S}_{2}$ n'est pas un rôle composite, mais un rôle simple de source assigné par le verbe inférieur. On observera d'ailleurs que, si l'on essaie de construire des contreparties "actives" à ce type d'énoncés, on obtient des suites apparemment grammaticales, mais que les anglophones considèrent comme inattestables : ?she couldn't make the maitre d' understand her /?...to make the public know his wartime work, etc. Par conséquent, seule demeure la relation d'implication: $\mathrm{S}_{1}$ agit de telle sorte que $\mathrm{X}$ le comprenne, entende, etc., et ce pour son propre bénéfice. 
De nombreux problèmes demeurent encore en suspens après cette étude ; nous pensons tout de même qu'une telle approche est à même de résoudre un certain nombre de questions, dont celle si délicate des substitutions possibles ou bloquées entre les quatre opérateurs. Il faudrait encore travailler dans plusieurs directions non exclusives, par exemple exploiter l'idée que certains opérateurs construisent un chemin nécessaire vers l'événement causé (c'est semble-t-il le cas de MAKE), d'autres un chemin contingent (c'est le cas de CAUSE); de là certains contrastes et blocages selon que l'événement causé soit ou non conforme aux attentes, souhaits ou craintes du causateur et/ou du causativé. On retrouve ici la notion de téléonomie, telle que l'utilise en particulier, la TOPE (voir entre autres les travaux de A. Deschamps), et qui nous semble une piste fructueuse pour affiner encore l'analyse.

C'est sans doute en suivant ces pistes qu'on arrivera à une analyse correcte des « causatifs lexicaux ", depuis très longtemps énigmatiques aux linguistes. Joseph and Mary stabled the horse ne pose aucun problème, mais *Joseph and Mary stabled the infant Jesus en pose un, qui est précisément de l'ordre de la téléonomie. John Wayne saddled his horse est commun, mais *the storm saddled the horse est rigoureusement exclu, même si la tempête a pour effet de faire envoler la selle qui se retrouverait par miracle dans la bonne position sur le cheval ${ }^{49}$. Et Sugar Ramos a bien frappé Davey Moore (I hit him, yes, it's true), mais, même si tous les ingrédients de la causalité directe sont réunis, il ne l'a pas pour autant tué...

\section{BIBLIOGRAPHIE}

ALSINA, A. (1992), 'On the argument structure of causatives', in Linguistic Inquiry, 23: 4, pp. 517-555

BOURQUIN, G. (1990), « Le statut linguistique de la cause », in RANAM XXIII, Université de

Strasbourg, pp. 13-32

COTTIER, E. (1991), "Les opérateurs causatifs de l'anglais, make, cause, have et get", Cahiers de Recherche en Grammaire Anglaise T.5, Gap, Ophrys

CROFT, W. (1991), Syntactic Categories and Grammatical Relations, Chicago, University of Chicago Press CULICOVER, P. \& R. JACKENDOFF (2005), Simpler Syntax, Oxford, O.U.P.

DAVIES, W. \& S. DUBINSKY (2004), The Grammar of Raising and Control, London, Blackwell

Dictionnaire Historique de la Langue Française, Robert (édition 1992)

FISCHER, O. (1990), Syntactic Change and Causation, Amsterdam, Amsterdam Studies in Generative Grammar

FISCHER, O. (1995), 'The distinction between bare and to-infinitival complements in late Middle

English, in Diachronica 12, pp. 1-30

FISCHER, O. (1996), 'Verbal complementation in early ME: how do the infinitives fit in?', in D. Britton (ed.), English Historical Syntax 1994, Amsterdam, John Benjamins, pp. 247-270 
FODOR, J. A. (1970) 'Three reasons for not deriving kill from cause to die.' in Linguistic Inquiry 1,1 : 429-438

GIVóN, T. (1993), English Grammar. A Function-Based Introduction, Amsterdam, John Benjamins HEINE, B. \& T. KUTEVA (2002), World Lexicon of Grammaticalization, Cambridge, C.U.P.

HigGinbotham, J. (2000), ‘Accomplishments', Proceedings of Glow in Asia II, Nagoya, Japan. Nagoya, Nanzan University, pp. 72-82.

HollmanN, W. (2003), Synchrony and Diachrony of English Periphrastic Causatives: a Cognitive Perspective, PhD. Thesis, University of Manchester

HOPPER, P. \& S. THOMPSON (1980), 'Transitivity in Grammar and Discourse', in Language 56, pp. 251-299. JACKENDOFF, R. (1990), Semantic Structures, Cambridge, MIT Press

KEMMER, S. \& A. VERHAGEN (1994), 'The grammar of causatives and the conceptual structure of events', in Cognitive Linguistics 5, pp. 115-156

KEMMER, s. (à par.), 'Causative Constructions and Cognitive Models: The English Make Causative' (paper presented at various conferences in 2000)

KHALIFA, J.-C. (2004), Syntaxe de l'anglais. Théories et pratique de l'énoncé complexe, Paris/Gap, Ophrys KHALIFA, J.-C. (à par.), « Verbes de perception et causatifs : une classe homogène ? », à paraître dans J.-C. Khalifa \& P. Miller, eds, Les Verbes de Perception KLAIMAN, M. H., (1991), Grammatical Voice, Cambridge, C.U.P.

LYONS, J. (1968), Introduction to Theoretical Linguistics, Cambridge, C.U.P.

MILLER, P. (2006), 'The position of English causative verbs in the object-control vs. raising-to-object dichotomy', communication au colloque « Constructions verbales et production du sens », Université de Besançon.

NEWMAN, J., ed. (1997), The Linguistics of Giving, Amsterdam, John Benjamins (Typological Studies in Language 36)

SKEAT, W. (1879-1882), An Etymological Dictionary of the English Language, Oxford, Clarendon

TALMY, L. (2001), Toward a Cognitive Semantics Vol. 1, Cambridge, MIT Press

TUGGY, D. (1997), 'Giving in Nawatl', in J. Newman, ed., pp. 35-65

VAN VALIN, R. D., \& R. J. LA POLLA (1997), Syntax. Structure, Meaning and Function, Cambridge, C.U.P.

VISSER, F. (1963-73), An Historical Syntax of the English Language, Vol. IV, Leiden, E. J. Brill

WATKINS, C., ed. (2000), The American Heritage Dictionary of Indo-European Roots, $2^{\text {nd }}$ Edition, Houghton Mifflin

YAMASHITA SMITH, T. (1998), 'How 'give' and 'receive' provide structure for more abstract notions: The case of benefactives, adversatives, causatives, and passives', Berkeley Linguistics Society 24, pp. 219-231.

\section{NOTES}

1. http://www.findagrave.com/cgi-bin/fg.cgi?page=gr\&GRid=4594 
2. Le présent article a été rédigé avant que l'auteur n'ait eu connaissance de l'étude de Philip Miller présentée au colloque «Constructions verbales et production du sens" (Université de Besançon), dont les conclusions recoupent en très grande partie les nôtres en ce qui concerne la syntaxe des causatifs.

3. Nous n'adoptons ce terme que pour pouvoir nommer cette construction, et sans trancher dans un débat qui a fait couler beaucoup d'encre et continue d'en faire couler. Depuis deux décennies au moins, les syntacticiens ont bien noté qu'il n'y avait pas véritablement de «montée » dans un tel cas, où la complémentation du V est IP et non $\mathrm{CP}$. Pour une présentation très exhaustive des enjeux théoriques de ces questions, voir Davies \& Dubinsky 2004.

4. Nous adoptons ici la présentation, très pédagogique, de Carnie 2002, qui donne simplement aux arguments propositionnels un rôle- $\theta$ «proposition ». On pourrait en l'occurrence l'appeler but, thème ou tout autre chose, du reste, cela ne changerait rien au raisonnement.

5. Point de vue que nous avons nous-même sinon défendu, au moins implicitement adopté dans Khalifa 2004. Bien évidemment, l'argument supplémentaire est forcément sujet syntaxique, morphologiquement au nominatif, mais cela ne préjuge en rien de son rôle sémantique.

6. Voir Khalifa 2004, Ch. 5.

7. Traduits en général par « causateur » et « causativé » (Cf. Cottier 1991)

8. Pour être précis, l'alternance n'est possible que si, comme dans l'exemple donné, le verbe inférieur est intransitif. Dans le cas contraire, seul NI est possible, puisque l'objet de ce verbe sera marqué par 0 , et qu'il existe en japonais comme dans la plupart des langues (y compris le français !) une contrainte syntaxique sur le double objet direct: on aura donc, pour reprendre notre exemple [3] supra, exemple qui nous a déjà beaucoup servi par le passé, [3'] John-GA ringo-o Mary-NI /*o tabe-sase-ta ( = John made / had Mary eat an apple).

9. Nous avons représenté leur positionnement par une zone floue plus étendue que celle de [11a][11b], en raison des différentes situations auxquelles, comme on vient de le montrer, ils sont susceptibles de renvoyer.

10. Cet espace, si réduit soit-il doit bien évidemment être cependant non nul ; il doit y avoir une relation de consécution entre les deux événements pour que l'interprétation cause 㽞 conséquence puisse se construire.

11. C'est dans ce cas aussi la connaissance partagée du monde qui va jouer : les monnaies n'étant pas susceptibles de cotation le week-end, c'est au plus tôt le lundi matin qu'a pu avoir lieu la chute de la livre sterling.

12. Voir en particulier Khalifa à paraître.

13. On trouve de tels exemples, bien que très rarement, en anglais moderne; ils sont presque invariablement des pastiches de styles anciens, en particulier bibliques.

14. On pourra nous objecter qu'il s'agit là de poésie, et que les contraintes métriques peuvent être responsables de l'alternance. Nous avons cependant retenu cet exemple pour des raisons de clarté, nous renvoyons le lecteur à Fischer 1995 pour d'autres exemples en prose tout aussi convaincants.

15. Exemple ${ }^{\circ} 1$ p. 1 de Fischer 1995. Il s'agit de l'histoire de Samson et Dalila.

16. Nous avions au départ adopté la terminologie "implicant»vs "impliqué ", mais elle soulevait quelques objections. Merci à Anne Ribot, étudiante d'agrégation à Poitiers, d'avoir suggéré " affectant » / " affecté », que nous avons finalement retenu.

17. Ici encore ( $c f$. Khalifa 2004, Ch.1 et 5), nous considérons que "bénéficiaire " subsume conséquences positives comme négatives de l'événement, donc nous ne ferons pas de distinction supplémentaire avec le « détrimentaire ».

18. C'est la raison pour laquelle nous avons schématisé/symbolisé ce trajet complexe par une flèche en boucle, mais il ne faut voir dans cette symbolisation aucune prétention à une quelconque représentation de schéma cognitif. 
19. Pour Skeat (1879-1882), la première étymologie est *mag, alors que G. Bourquin (1990) privilégie le lien à match. On trouve les deux à égalité chez $\mathrm{C}$. Watkins.

20. "'cause' [...] est emprunté au latin causa, d'origine inconnue (prélatin ou emprunté)» (Robert Historique de la Langue Française).

21. Nous ignorons en particulier la distinction entre « $\mathrm{v}$ » et « $\mathrm{V}$ », pour ne pas multiplier les projections.

22. Ce qui n'est pas sans soulever de problèmes théoriques énormes sur le statut de cet opérateur; là encore, nous en sommes conscient, mais passons rapidement pour ne pas alourdir le propos.

23. Le Khmer, le Thai, le Vietnamien, entre autres (Heine \& Kuteva, Yamashita Smith) ; Tuggy 1997 mentionne que, parmi les nombreux suffixes dérivationnels du Nahuatl, beaucoup forment des causatifs ou applicatifs, et 'a number of these causativized and applicativized stems express meanings very close to GIVE.' Nous évitons, pour ne pas allonger démesurément cette étude, de citer les exemples dans ces langues.

24. Emprunté à Heine \& Kuteva.

25. Le trajet initial $\left(\mathrm{S}_{1}\right.$ vers $\left.\mathrm{S}_{2}\right)$, mis à l'arrière-plan, est davantage présupposé que posé, comme tout retour présuppose un aller.

26. Exemple emprunté à Klaiman 1991 (nº 12 p. 24).

27. Pour être plus précis, il faudrait parler d'inergatifs, puisque, sous réserve d'inventaire, on n'y trouve que des intransitifs à sujet agentif.

28. Il serait d'ailleurs plus prudent et plus exact, en toute rigueur, de parler d'un rôle agentif et d'un rôle patientif ; des oppositions plus globales comme les proto-rôles à la Dowty, ou encore les macro-rôles (actor / undergoer chez Van Valin \& La Polla) nous semblent en effet plus opératoires ici : dans le réfléchi par exemple, c'est bien à la fois comme agent et patient qu'il faut analyser le sujet. Il est d'autres situations où le rôle dominant sera plutôt expérient qu'agent, etc. 29. Si l'on dit parfois (y compris, comme nous le signale C. Rivière (c. p.), dans des ouvrages pédagogiques) que des exemples au passif avec CAUSE comme en [21] sont difficilement attestables (voire inattestables), c'est à notre sens tout simplement à cause de la rareté (cf. tableau n ${ }^{\circ} 1$ ) de la construction en CAUSE elle-même. Nous-même avons eu parfois du mal à en faire construire par des anglophones à partir de structures actives, certains déclarant la transformation impossible...

30. Nous avons utilisé le logiciel SCP (version 4.0.8.), mis au point par Alan Reed, et disponible en freeware.

31. Remarque faite, entre autres, par C. Rivière, Journées de l'ALAES sur les causatives, Charles V, $21 / 1 / 2005$.

32. Nous avons collecté de tels exemples autant avec imbriquée à l'actif qu'au passif. Il faudrait cependant faire un travail beaucoup plus détaillé encore pour étudier les fortes contraintes, car à l'actif il semble (cf. [24]) que l'on ne puisse trouver que des intransitifs.

33. Ch. 5 , § 3.5.1., ex. [76"']. Nous n'avions pas encore, à l'époque pourtant récente des révisions pour la nouvelle édition, commencé à fouiller les corpus pour la présente étude.

34. Là encore, il y a tout un domaine de recherche fine : les seuls exemples authentiques du schéma de fusion que nous avons pu trouver (pour l'instant) avec MAKE sont soit make appear, soit make happen. Il semble se dégager un ensemble de contraintes communes, mais nous ne pouvons encore nous prononcer avec précision.

35. Nous avons, alors que la présente étude était en cours, appris l'existence de l'étude encore inédite de Suzanne Kemmer (Kemmer à par.), que nous remercions ici pour l'avoir mise à notre disposition. Ses résultats statistiques, portant sur 201 occurrences extraites du B.N.C., sont à quelques décimales près les mêmes que les nôtres. Les exemples donnés ici sont ceux que nous avons nous-même relevés dans notre corpus de fiction.

36. "The predicates found can be roughly classified heuristically into the following types: 1) physiological response (itch, shiver, cringe, burp); 2) emotive response with a physical aspect (laugh, cry, sigh, smile; 
3) emotive response with a mental aspect (feel good, feel old, feel special, suffer); and 4) a mental event involving higher mental processing (feel that, think, believe)" (Kemmer, op. cit.). Il ne faut voir aucune contradiction dans le fait que, dans John made me laugh, John peut parfaitement n'avoir strictement rien fait. C'est bien le locuteur qui reconstruit une causation qui fait sens pour lui, mais qui est purement du déductif a posteriori; il n'empêche que cette causation est bel et bien construite comme directe.

37. On retrouve ici une autre analogie, celle des évidentiels, que nous avions déjà abordée dans une étude sur les verbes de perception, mais nous ne pousserons pas plus loin ce parallèle ici, nous réservant d'y revenir dans une prochaine étude..

38. Une note méthodologique s'impose : les 157 occurrences de MAKE sur lesquelles nous avons travaillé pour établir le tableau $n^{\circ} 2$ sont bien les mêmes que celles que l'on retrouve dans le tableau $n^{\circ} 1$, c'est-à-dire celles qui viennent de notre propre corpus de fiction. Nous avons estimé, compte tenu du fait que S. Kemmer, avec 201 exemples tirés du B.N.C., arrivait très exactement aux mêmes résultats que les nôtres, que nous pouvions nous en tenir là. En revanche, il nous a fallu nous tourner vers le B.N.C. pour extraire un nombre suffisant d'occurrences de CAUSE, GET et HAVE, les échantillons obtenus dans le tableau $n^{\circ} 1$ (respectivement 4,30 et 20) étant beaucoup trop réduits pour être significatifs.

39. «Agent» et "patient» dans les cas prototypiques, bien entendu il peut s'en présenter d'autres, mais cela ne change rien au raisonnement.

40. Nous avions repris dans Khalifa 2004 telle quelle cette analyse, pour laquelle nous n'avions pas à l'époque d'explication convaincante.

41. Bien entendu, on ne prendra pas «complémentaire » ici au sens que lui donne la TOPE en matière de domaines notionnels.

42. Cf. Cottier 1991 op. cit., Khalifa 2004 op. cit. Au stade actuel de notre réflexion, nous sommes davantage tenté de voir dans cette valeur un effet pragmatique et non une composante primitive de GET : HAVE en effet renvoie, comme on le sait, à un état stable, et GET au passage de frontière qui mène à cet état. Le fait même qu'il y ait franchissement, dans une relation de type causatif, suffit souvent à induire cet effet.

43. Nous n'insisterons pas sur les analyses standard de V-ING, suffisamment connues pour ne pas s'y attarder. Nous faisons ici allusion à l'effet de « dilatation » souvent mis en évidence pour $B E+$ $I N G$, qui au fond revient à un " arrêt sur images » et par conséquent à un état temporaire.

44. La négation joue un rôle crucial dans cet exemple : il n'y a justement pas eu validation, HAVE a donc l'avantage de rester neutre.

45. Un peu de contexte pour bien comprendre cet exemple : la locutrice est la mère du narrateur, et Rex est son père ; ils sont séparés depuis quelques mois, mais le père a invité son ex-épouse à l'accompagner à un dîner.

46. Ce point sera repris dans une étude à paraître sur les rapports entre causatifs et verbes de perception.

47. Ch. 5, § 3.5.1.

48. Ce dernier exemple était déjà dans Khalifa 2004 (ex. [88])

49. Ces exemples sont empruntés à Higginbotham 2000. 


\section{RÉSUMÉS}

Le present article est une tentative de formalisation du micro-système formé par les 4 opérateurs causatifs de l'anglais contemporain, (make, have, cause et get). D'un point de vue syntaxique, ces constructions sont ambiguës entre contrôle et montée, ce qui soulève le problème des rôles sémantiques associés aux arguments agent et thème. Notre hypothèse est que ces derniers sont à analyser comme des rôles composites, ce qui nous amène, dans une deuxième partie, à proposer une cartographie des 4 verbes, sur un modèle de quadrants définis par deux axes : causation directe vs. Indirecte, et causateur affectant vs. affecté. La dernière partie met les hypothèses avancées à l'épreuve d'une vaste étude de corpus, et montre comment divers effets de sens peuvent être prédits à partir du modèle.

This paper is an attempt to formalize the micro-system of the 4 causative verbs (i.e. make, have, cause and get) in modern English. The syntax of causative constructions is ambiguous between control and raising, which raises the problem of the semantic roles of both agent and theme arguments. We argue that those are to be analyzed as composite roles, which in turn leads us to propose a quadrant analysis of the 4 verbs along the two axes of direct vs. indirect causation and involved vs. involving causer. In the $3^{\text {rd }}$ part, a vast corpus analysis shows how the theoretical model may predict and explain the different discourse effects.

\section{INDEX}

Mots-clés : constructions causatives, causateur, causativé, étude de corpus, rôles sémantiques

Keywords : causative constructions, causer, causee, semantic roles, corpus study

\section{AUTEUR}

\section{JEAN-CHARLES KHALIFA}

Université de Poitiers, Laboratoire FORELL 